\title{
Experimental Validation of MCNP Models for PINS Shadow Shield Mockups
}

D. Lee

B. Bucher

K. Krebs

E. Seabury

J. Wharton

April 2019

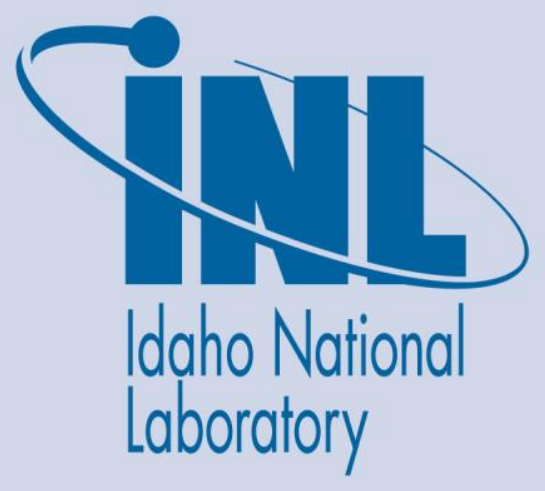

The INL is a U.S. Department of Energy National Laboratory operated by Battelle Energy Alliance 


\section{DISCLAIMER}

This information was prepared as an account of work sponsored by an agency of the U.S. Government. Neither the U.S. Government nor any agency thereof, nor any of their employees, makes any warranty, expressed or implied, or assumes any legal liability or responsibility for the accuracy, completeness, or usefulness, of any information, apparatus, product, or process disclosed, or represents that its use would not infringe privately owned rights. References herein to any specific commercial product, process, or service by trade name, trade mark, manufacturer, or otherwise, does not necessarily constitute or imply its endorsement, recommendation, or favoring by the U.S. Government or any agency thereof. The views and opinions of authors expressed herein do not necessarily state or reflect those of the U.S. Government or any agency thereof. 


\title{
Experimental Validation of MCNP Models for PINS Shadow Shield Mockups
}

\author{
D. Lee \\ B. Bucher \\ K. Krebs \\ E. Seabury \\ J. Wharton
}

April 2019

\author{
Idaho National Laboratory \\ Nuclear Nonproliferation Division \\ Idaho Falls, Idaho 83415
}

http://www.inl.gov

Prepared for the

U.S. Department of Energy

Office of National Nuclear Security Administration

Under DOE Idaho Operations Office

Contract DE-AC07-05ID14517 



\begin{abstract}
MCNP has been regarded as an indispensable tool in development of radiation detection systems, and it is cost effective, thanks to advances in computing power, to simulate various conceptual designs without even fabricating them. Development of various PINS systems have also benefited from MCNP modeling and simulation. MCNP models of PINS systems have been frequently used to understand detector responses and to fill the gap in experimental data. Recently, a backpack version PINS system was announced to be developed. When the neutron generator and the HPGe detector are built by external vendors, the shadow shield is the component to be designed to maximize the performance while meeting the sponsor's requirements. In order to utilize MCNP modeling and simulation to find the best specification of the shadow shield, a campaign of PINS lab measurements with a few shadow shield mockups has been conducted to validate MCNP simulation results. This study is intended to validate MCNP simulations and models in preparation of another more extensive simulation study. Validation of neutron flux is also discussed later in this report even though neutron counts were not measured experimentally.
\end{abstract}




\section{CONTENTS}

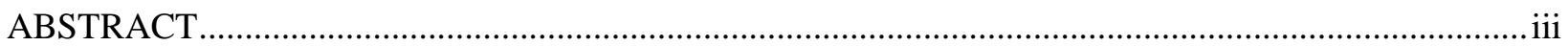

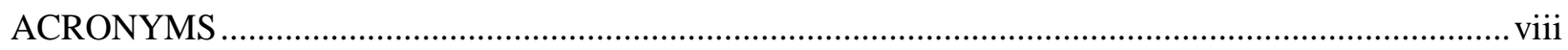

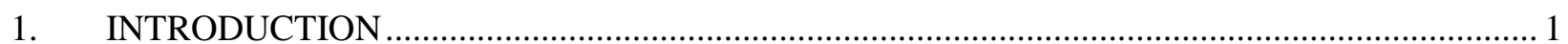

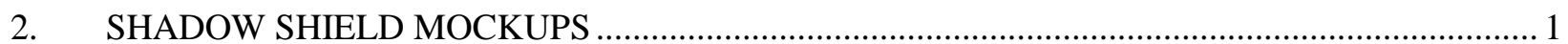

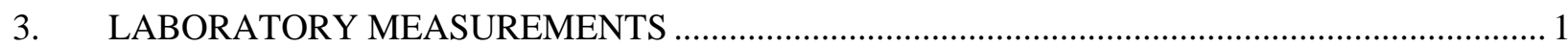

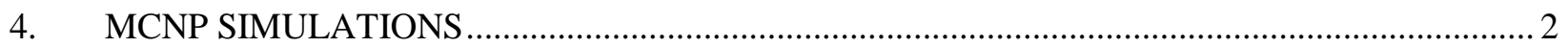

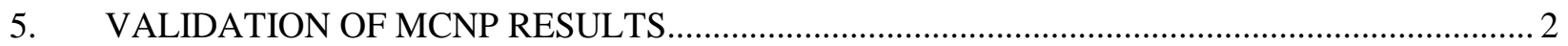

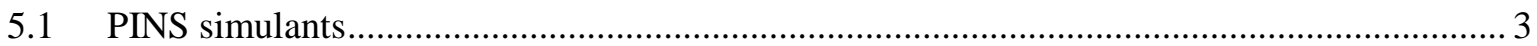

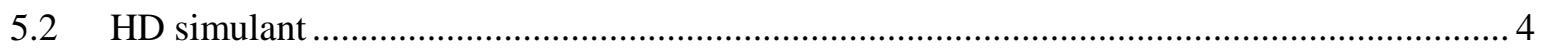

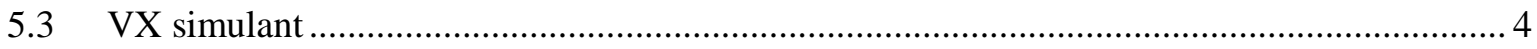

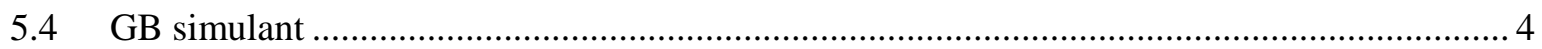

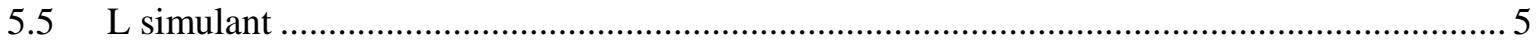

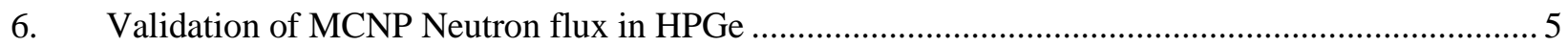

7. Summary

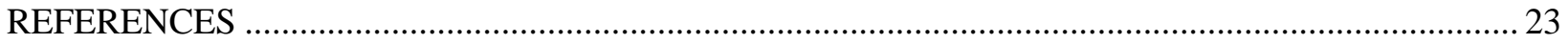

\section{FIGURES}

Figure 1. Six configurations of the shadow shield used in the experiments .......................................... 7

Figure 2. PINS laboratory setup with a P385 D-D generator and a simulant in place.............................. 10

Figure 3. (Top) averaged dead times of the measured spectra are plotted as a function of the tungsten block volume. (Bottom) averaged spectral qualities of the measured spectra are plotted as a function of the tungsten block volume ......................................................... 10

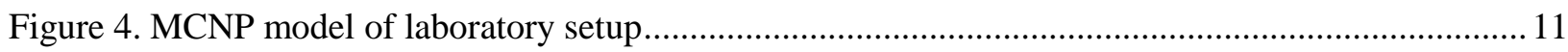

Figure 5. MCNP models of six unique shadow shield configurations................................................... 11

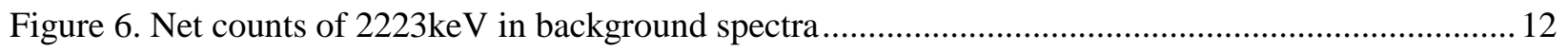

Figure 7. (Top) changes in net counts for various HD simulant densities. (Bottom) changes in net counts for various VX simulant densities

Figure 8. Net counts of gamma-ray peaks from key elements in The HD simulant, assuming The HD simulant's density is $1.434 \mathrm{~g} / \mathrm{cm}^{3}$.

Figure 9. Net counts of gamma-ray peaks from key elements in The HD simulant for various The HD simulant's densities of $1.434 \mathrm{~g} / \mathrm{cm}^{3}, 1.234 \mathrm{~g} / \mathrm{cm}^{3}$ and $1.034 \mathrm{~g} / \mathrm{cm}^{3}$. 
Figure 10. Net counts of gamma-ray peaks from key elements in the VX simulant ................................ 16

Figure 11. Net counts of gamma-ray peaks from key elements in the GB simulant ............................... 17

Figure 12. Net counts of gamma-ray peaks from key elements in the $\mathrm{L}$ simulant .................................. 18

Figure 13. An example of HPGe spectrum from the measurements....................................................... 19

Figure 14. MCNP neutron fluxes of six configurations with no simulant .............................................. 19

Figure 15. (Top) net counts of $139.7 \mathrm{keV}$ from ${ }^{74} \mathrm{Ge}(\mathrm{n}, \gamma)$ reaction were compared to $\mathrm{N}_{\text {total }}$. (Bottom) net counts of $689.6 \mathrm{keV}$ from ${ }^{72} \mathrm{Ge}\left(\mathrm{n}, \mathrm{n}^{\prime} \gamma\right)$ reaction were compared to $\mathrm{N}_{\text {fast }} \ldots \ldots \ldots \ldots . . . . .20$

Figure 16. (Left column) net counts of $139.7 \mathrm{keV}$ were compared to $\mathrm{N}_{\text {total }}$ for each simulant.

(Right column) net counts of $689.6 \mathrm{keV}$ were compared to $\mathrm{N}_{\text {fast }}$ for each simulant .................21

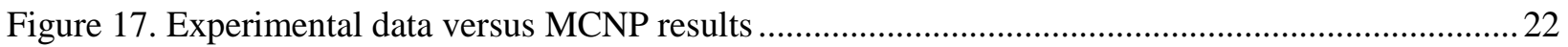

\section{TABLES}

Table 1. Summary of experimental data analyzed in this study ............................................................. 8

Table 2. Measured and simulated net counts of hydrogen in background spectra..................................... 12

Table 3. Measure and simulated net counts of chlorine, hydrogen and sulfur for The HD simulant .......... 14

Table 4. Measured and simulated net counts of phosphorus and hydrogen for the VX simulant............... 16

Table 5. Measured and simulated net counts of phosphorus and hydrogen for the GB simulant ............... 17

Table 6. Measured and simulated net counts of chlorine, hydrogen and sulfur for the L simulant............ 18

Table 7. Summary of germanium 139.7 and $689.6 \mathrm{keV}$ net counts from the measured background spectra. 


\section{ACRONYMS}

$\begin{array}{ll}\text { D-D } & \text { Deuterium-deuterium } \\ \text { EMG } & \text { Exponentially modified Gaussian } \\ \text { GB } & \text { Sarin nerve agent } \\ \text { GEB } & \text { Gaussian Energy Broadening } \\ \text { HD } & \text { Sulfur mustard blister agent } \\ \text { HDPE } & \text { High density polyethylene } \\ \text { HPGe } & \text { High purity germanium } \\ \text { INL } & \text { Idaho National Laboratory } \\ \text { L } & \text { Lewisite blister agent } \\ \text { MCNP } & \text { Monte Carlo n-particle } \\ \text { PINS } & \text { Portable Isotopic Neutron Spectroscopy } \\ \text { SSR } & \text { The sum of squared residuals } \\ \text { VX } & \text { Nerve agent }\end{array}$




\section{Experimental Validation of MCNP Models for PINS Shadow Shield Mockups}

\section{INTRODUCTION}

MCNP has been regarded as an indispensable tool in development of radiation detection systems, and it is cost effective, thanks to advances in computing power, to simulate various conceptual designs without even fabricating them. Development of various PINS systems have also benefited from MCNP modeling and simulation. MCNP models of PINS systems have been frequently used to understand detector responses and to fill the gap in experimental data. Recently, a backpack version PINS system was announced to be developed. When the neutron generator and the HPGe detector are built by external vendors, the shadow shield is the component to be designed to maximize the performance while meeting the sponsor's requirements. It is obvious for us to rely on MCNP modeling and simulation to find the best specification of the shadow shield. Therefore, a campaign of PINS lab measurements with a few shadow shield mockups has been conducted to be compared to MCNP simulation results. This study is intended to validate MCNP simulations and models in preparation of another more extensive simulation study. This study is mainly focused on comparing gamma-ray responses of the HPGe detector between MCNP and experimental results, but validation of neutron flux is also discussed later in this report even though neutron counts were not measured experimentally.

\section{SHADOW SHIELD MOCKUPS}

Six different configurations of the shadow shield were prepared by combining tungsten blocks with polyethylene blocks in preparation of an experimental campaign. Three existing tungsten blocks in the lab were used to save time and cost, and high density polyethylene (HDPE) blocks were either newly fabricated to match the dimensions of the tungsten blocks or picked up from the stock if their sizes match the tungsten blocks. Existing tungsten blocks of three different dimensions were selected to avoid making new ones: their dimensions ( $\mathrm{L} \times \mathrm{W} \times \mathrm{H}$ ) are 3" x 2-13/16" x 2-13/16" (hereinafter referred to as "medium"), 1-1/8" x 2-13/16" x 2-13/16" (hereinafter referred to as "small") and 2" x 4" x 4" (hereinafter referred to as "large") as shown in Figure 1. Two different shapes of HDPE blocks were used: truncated wedge (hereinafter referred to as "wedge") and truncated pyramid (hereinafter referred to as "pyramid"). An existing HDPE pyramid block was used to build the configuration \#1 and \#2 while new HDPE blocks were fabricated to build the configurations \#3 through \#6. The shadow shields were oriented to place the HDPE blocks close to the generator and the tungsten blocks close to the HPGe detector.

\section{LABORATORY MEASUREMENTS}

A P385 D-D neutron generator (operated at $130 \mathrm{kV}$ and $70 \mu \mathrm{A}$ ) and a nitrogen cooled HPGe detector (system ID P09) were used as fixtures in a PINS setup assembled for this study. Then, a total of six unique shadow shield configurations were tested in turn with PINS simulants. Figure 2 shows the experimental PINS setup coupled to various shadow shield mockups in the PINS laboratory. Four different PINS simulant cylinders were used in the laboratory measurements: HD, VX, GB and L. As 
usual, a background spectrum was collected for 1000-second as the first measurement of each day, and a spectrum for each simulant was collected for 3000-second one after another. This data collection routine was repeated three times to yield three independent sets of gamma-ray spectra for each combination of one shadow shield configuration with one type of simulant. As a result, a total of 72 PINS simulant spectra (6 shadow shield configurations x 4 simulants x 3 measurements) and a total of 12 background spectra (two per configuration) were saved to be analyzed at the end of the campaign. Table 1 summarizes a list of the laboratory measurements included in this study. Figure 3 shows a plot of the dead times and the spectral quality factors of the measured spectra. The tungsten block's volume is only one of the factors to describe the distribution of dead times, but a tungsten block with optimal width and thickness could stop excessive gamma-rays in addition to being a good neutron shield to the HPGe detector. Dead times were reported below 35\% for all cases to be considered normal. Figure 3 also shows a plot of the spectral qualities of the measured spectra, but it is not straightforward to explain their behaviors as a simple function of the tungsten volume. The spectral quality in PINS+ is calculated by two high energy iron peaks so the presence of a simulant cylinder and the overall geometry would be more relevant factors to explain their behaviors. It is speculated that configuration \#5 and \#6 might have scattered iron's $7631 \mathrm{keV}$ and $7645 \mathrm{keV}$ gamma-rays away from the HPGe detector due to their larger areas. Nonetheless, all measured spectra were considered normal with spectral quality factors above $85 \%$.

\section{MCNP SIMULATIONS}

Each MCNP simulation was run with $1.5 \times 10^{10} \mathrm{D}$-D neutrons emitted isotropically, and the pulse height tally (F8 tally) was treated with Gaussian energy broadening (GEB) to mimic HPGe detector responses in a real PINS system. Then, the tally information in the MCNP output file was converted to a MAESTRO ${ }^{\circledR}$ $\mathrm{CHN}$ format file. A special version of PINS+ was used to analyze the CHN file as if it was collected from a normal laboratory measurement. A total of 30 simulations were run to match all experimental data (6 shadow shield configurations x 4 simulants +6 backgrounds). Figure 4 shows an MCNP model of the setup with one shadow shield configuration. The neutron generator controller and the blue steel cart under the setup shown in Figure 2 were omitted to make MCNP models less complicated. Figure 5 shows MCNP models of the six shadow shield mockups with the exact dimensions specified in Figure 1. Chemical compositions of the HD, VX, GB and L simulants were adopted from their corresponding lists of mixture ingredients, but their final densities were not well documented when the cylinders were filled and sealed. Therefore, the densities of the four simulants used in MCNP simulations were simply calculated to our best knowledge based on the formulae. Additionally, it should be noted that these simulants in MCNP simulations were treated as homogeneous compounds while actual simulants are more likely to be heterogeneous mixtures.

\section{VALIDATION OF MCNP RESULTS}

Chlorine, hydrogen and sulfur peaks were used to compare MCNP results to experimental data for the HD simulant: 1764, 1952 and $1960 \mathrm{keV}$ from chlorine, $2223 \mathrm{keV}$ from hydrogen and $2230 \mathrm{keV}$ from sulfur. Phosphorus and hydrogen peaks were used for the VX and the GB simulant: 1266keV from phosphorus and $2223 \mathrm{keV}$ from hydrogen. Phosphorus's $5420 \mathrm{keV}$ peak was not included because MCNP was unable to reproduce $5420 \mathrm{keV}$ gamma-rays due to lack of data in its internal library. Chlorine, hydrogen and arsenic peaks were used for the L simulant: 1764, 1952 and $1960 \mathrm{keV}$ from chlorine, 2223keV from 
hydrogen and $279 \mathrm{keV}$ from arsenic. Net counts for these peaks were extracted from the analysis files created by PINS+.

Table 2 shows net counts of hydrogen peak at $2223 \mathrm{keV}$ in the background spectra, and Figure 6 shows a plot of the data in Table 2 to compare MCNP results to experimental data. All MCNP results were multiplied by $6.56 \times 10^{9}$, a scale factor determined from the best-fit between MCNP results and experimental data. The sum of squared residuals (SSR) value was minimized to find the best-fit in the hydrogen $2223 \mathrm{keV}$ plot of Figure 6 . As a measure to validated MCNP results against experimental data, the correlation coefficient, $R$, was adopted to quantify how well MCNP models reproduce experimental data. A correlation coefficient of 0.997 was obtained between the paired data sets in Table 2, which implies that experimental data are predictable from MCNP models with a highly linear relationship. Overall, the MCNP simulations with no simulant represent the actual detector responses very well as shown in Figure 6.

\subsection{PINS simulants}

In MCNP simulations, simulant density is an important physical property to be provided by users to replicate detector responses in the experimental setup. Unfortunately, the densities of the HD, VX, GB and The L simulants were not accurately known so that each simulant's density had to be estimated based on its formula for the composite mixture as discussed above. In order to assess the effect of density on MCNP results, various densities of the HD simulant were tested in the MCNP model of configuration \#6. The estimated density was calculated to be $1.434 \mathrm{~g} / \mathrm{cm}^{3}$ from its formula by default, and three different values of density were substituted in the same MCNP model. Figure 7 shows changes in net counts of the key gamma-ray peaks when the simulant density was varied from 1.034 to $1.834 \mathrm{~g} / \mathrm{cm}^{3}$ in increments of $0.200 \mathrm{~g} / \mathrm{cm}^{3}$. As expected, thermal neutron capture peaks at 1952,1960 and $2223 \mathrm{keV}$ are more sensitive to simulant density than neutron inelastic peaks at 1764 and $2230 \mathrm{keV}$. These results show that D-D neutrons are more effectively slowed down with denser materials to increase thermal neutron capture reactions. Fast neutron induced inelastic gamma-rays are nearly insensitive to simulant density. For the VX simulant, the density of $1.378 \mathrm{~g} / \mathrm{cm}^{3}$ was estimated from its formula by default. Two additional densities of 1.678 and $1.078 \mathrm{~g} / \mathrm{cm}^{3}$ were tested to observe changes in net counts of 1266 and $2223 \mathrm{keV}$ as shown in Figure 7. Again, net counts at $1266 \mathrm{keV}$ from neutron inelastic scattering are nearly identical regardless of simulant density while net counts at $2223 \mathrm{keV}$ from thermal neutron capture are linearly proportional to density.

These results suggest that chlorine $1764 \mathrm{keV}$ or phosphorus $1266 \mathrm{keV}$ peaks are more reliable than thermal neutron capture peaks because simulant densities are less accurate. Therefore, a scale factor for each simulant was determined by fitting the $1764 \mathrm{keV}$ or $1266 \mathrm{keV}$ net counts of all six configurations between MCNP results and experimental data. This scale factor was applied to MCNP results of the same simulant. Strong positive correlation between MCNP results and experimental data must be confirmed in order to predict performance of any new designs based on MCNP simulations. Table 3 through 6 summarizes net counts of full energy peaks from key elements in four simulants, respectively. As discussed above, three independent 3000-second spectra were collected for each configuration with one type of simulant. Therefore, nominal net counts for experiments in Table 3 through 6 were taken from average values of three laboratory measurements. MCNP results presented in Table 3 through 6 were multiplied by the same scale factor determined for each simulant. Figure 8 through 12 show plots of the data summarized in Table 3 through 6. 


\subsection{HD simulant}

Table 3 summarizes MCNP results and experimental data for The HD simulant. Chlorine 1764, 1952 and $1960 \mathrm{keV}$, hydrogen $2223 \mathrm{keV}$ and sulfur $2230 \mathrm{keV}$ are characteristic gamma-ray peaks to validate MCNP results for The HD simulant. Density of $1.434 \mathrm{~g} / \mathrm{cm}^{3}$ was used in MCNP models as discussed above. Figure 8 shows plots of the net counts listed in Table 3. All MCNP results were multiplied by $5.43 \times 10^{9}$, a scale factor determined from the best-fit between MCNP results and experimental data. The sum of squared residuals (SSR) value was minimized to find the best-fit in the chlorine $1764 \mathrm{keV}$ plot of Figure 8. When MCNP results are scaled to match chlorine $1764 \mathrm{keV}$ peaks, the net counts from MCNP results are overestimated for the thermal neutron capture peaks. One possible explanation for these discrepancies is that The HD simulant's density of $1.434 \mathrm{~g} / \mathrm{cm}^{3}$ in MCNP models might be higher than the true density value as shown in Figure 7. Changes in the net counts of thermal neutron capture peaks are shown in Figure 9 if lower density values are used instead of the default one, and it is clear that density is one factor to be adjusted in MCNP models to suppress excessive thermal neutron capture peaks in MCNP results. Overall, MCNP models were capable of representing the behaviors of experimental data although there is still uncertainty in The HD simulant's density. A correlation coefficient of 0.814 shows that actual detector responses could be predicted by MCNP results with a linear relationship. MCNP results with low densities were reported to have better correlation coefficients: 0.876 for $1.234 \mathrm{~g} / \mathrm{cm}^{3}$ and 0.938 for $1.034 \mathrm{~g} / \mathrm{cm}^{3}$.

\subsection{VX simulant}

Table 4 summarizes MCNP results and experimental data for the VX simulant. Phosphorus 1266keV and hydrogen $2223 \mathrm{keV}$ were used to validate MCNP models for the VX simulant. Density of $1.378 \mathrm{~g} / \mathrm{cm}^{3}$ was used in MCNP models initially. Figure 10 shows plots of the net counts listed in Table 4. All MCNP results were multiplied by $9.68 \times 10^{9}$, a scale factor determined from the best-fit between MCNP results and experimental data. The best-fit was obtained by minimizing the SSR value for the phosphorus 1266keV peaks as shown in Figure 10.

When MCNP results are scaled to match phosphorus $1266 \mathrm{keV}$ peaks, the net counts from MCNP results are overestimated for hydrogen thermal neutron capture peaks. One possible explanation for these discrepancies is that the VX simulant's density of $1.378 \mathrm{~g} / \mathrm{cm}^{3}$ in MCNP models might be a bit higher than the true value as explained in Figure 7. Overall, MCNP models were capable of representing the behaviors of experimental data although there is still uncertainty in the VX simulant's density. A correlation coefficient of 0.983 was calculated between MCNP results and experimental data.

\subsection{GB simulant}

Table 5 summarizes MCNP and experimental data for the GB simulant. Phosphorus $1266 \mathrm{keV}$ and hydrogen $2223 \mathrm{keV}$ were used to validate MCNP models for the GB simulant. Density of $1.820 \mathrm{~g} / \mathrm{cm}^{3}$ was used in MCNP models initially. Figure 11 shows plots of the net counts listed in Table 5. All MCNP results were multiplied by $6.04 \times 10^{9}$, a scale factor determined from the best-fit between MCNP results and experimental data. The best-fit was obtained by minimizing the SSR value for the phosphorus 1266keV peaks as shown in Figure 11. 
When MCNP results are scaled to match phosphorus $1266 \mathrm{keV}$ peaks, the net counts from the MCNP results are a bit overestimated for hydrogen thermal neutron capture peaks. It seems that the GB simulant's density of $1.820 \mathrm{~g} / \mathrm{cm}^{3}$ in MCNP models is relatively close to the true value. Overall, MCNP models were capable of representing the behaviors of experimental data although there is still uncertainty in the GB simulant's density. A correlation coefficient of 0.974 was calculated between MCNP results and experimental data.

\subsection{L simulant}

Table 6 summarizes MCNP and experimental data for the L simulant. Chlorine 1764, 1952 and 1960keV, hydrogen $2223 \mathrm{keV}$ and arsenic $279 \mathrm{keV}$ were selected as key gamma-ray peaks to validate MCNP results for the L simulant. Density of $1.895 \mathrm{~g} / \mathrm{cm}^{3}$ was used in the MCNP simulations initially. Figure 12 shows plots of the net counts listed in Table 6. All MCNP results were multiplied by $4.49 \times 10^{9}$, a scale factor determined from the best-fit between MCNP and experimental results. The best-fit was obtained by minimizing the SSR value for the chlorine $1764 \mathrm{keV}$ peaks as shown in Figure 12.

When MCNP results are scaled to match chlorine $1764 \mathrm{keV}$ peaks, the net counts from the MCNP simulations are underestimated for thermal neutron capture peaks. MCNP results are noticeably low for arsenic $279 \mathrm{keV}$ peaks by a factor of two for all six configurations. One possible explanation for these discrepancies is that the L simulant's physical properties in MCNP models might be inaccurate. Overall, MCNP models were capable of representing the behaviors of experimental data although there is still uncertainty in the L simulant's density. A correlation coefficient of 0.968 was calculated between MCNP results and experimental data.

\section{Validation of MCNP Neutron flux in HPGe}

Shadow shields in PINS systems also prevent high energy neutrons from directly hitting the HPGe crystal in order to minimize neutron damage. Therefore, neutron energy distribution in the HPGe crystal is another important factor to be validated in MCNP results, but neutron counts were not measured in this experimental campaign. Moreover, current version of MCNP code is capable of simulating only few neutron induced gamma-rays from the germanium isotopes. Therefore, direct comparison between experimental and simulated gamma-ray spectra is inappropriate to validate MCNP neutron results. Neutron fluxes in the HPGe crystal, however, could be indirectly estimated by correlating germanium peaks in experimental spectra with MCNP neutron tallies. As shown in Figure 13, 139.7keV gamma-ray peak from ${ }^{74} \mathrm{Ge}(\mathrm{n}, \gamma)$ reaction are relatively well isolated to be analyzed, and this peak has been used to estimate thermal neutron flux in a previous study [1]. Also, $689.6 \mathrm{keV}$ peak with a high energy tail is populated by ${ }^{72} \mathrm{Ge}(\mathrm{n}, \mathrm{n}$ ' $\gamma)$ reaction by fast neutrons. It was speculated that there would be positive correlations between the intensities of these peaks and the MCNP neutron fluxes, and it has been shown that MCNP simulations without simulants reproduced actual detector responses very well. Therefore, background spectra from all six configurations (two per each configuration) were used to analyze germanium peaks. $139.7 \mathrm{keV}$ full energy peak was manually fitted with a Gaussian function to extract net counts since this peak was not included in PINS+ analysis. $689.6 \mathrm{keV}$ peak had to be manually fitted with an exponentially modified Gaussian (EMG) function due to its high energy tail.

Average net counts of 139.7 and $689.6 \mathrm{keV}$ peaks from each configuration's two background spectra are summarized in Table 7. As shown in Figure $14,{ }^{74} \mathrm{Ge}(\mathrm{n}, \gamma)$ reaction cross-section extends over the entire 
energy range although thermal neutrons contribute significantly more than fast neutrons. ${ }^{72} \mathrm{Ge}(\mathrm{n}, \mathrm{n}$ ' $\gamma$ ) reaction is induced by fast neutrons above $0.7 \mathrm{MeV}$. Hence, F4 neutron flux tally assigned to the HPGe detector cell in MCNP models was used to derive the total number of neutrons, $N_{\text {total }}$, over the entire energy range up to $5 \mathrm{MeV}$ and the total number of fast neutrons, $N_{\text {fast }}$, above $1 \mathrm{MeV} . N_{\text {total }}$ and $N_{\text {fast }}$ are also summarized in Table 7 . Figure 15 shows a plot of the germanium $139.7 \mathrm{keV}$ counts along with $N_{\text {total }}$ and another plot of the germanium $689.6 \mathrm{keV}$ counts with $N_{\text {fast }}$. Both plots show strong positive correlations between the germanium counts and the $N_{\text {total }}$ and $N_{\text {fast }}$. The same analysis was applied to the spectra with simulants, and Figure 16 shows plots of $139.7 \mathrm{keV}$ peak counts compared to $N_{\text {total }}$ (left column) and $689.6 \mathrm{keV}$ peak counts compared to $N_{\text {fast }}$ (right column) for four simulants. A correlation coefficient between the pair of data sets in each plot is shown as a quantitative measure to validate that MCNP models replicate neutron fluxes for various shadow shield configurations correspondingly.

\section{Summary}

MCNP models of six shadow shield configurations were validated against experimental data collected in the PINS laboratory. HPGe gamma-ray spectra were used to compare net counts of characteristic gammaray peaks in four simulants and background runs. Figure 17 shows plots of MCNP net counts versus experimental net counts for background and four simulants, respectively. MCNP net counts with simulants were normalized to match the net counts of either chlorine $1764 \mathrm{keV}$ or phosphorus $1266 \mathrm{keV}$ in experimental data. As shown in Figure 17, there is a positive linear relationship between MCNP results and experimental data. This study also reveals that simulant mixture's density and non-homogeneity is likely to be one cause of discrepancies between MCNP results and experimental data. Two plots of The HD simulant with different densities in Figure 17show that simulant's density in the models has a significant impact on MCNP results. In conclusion, a strong positive correlation between MCNP results and experimental data implies that MCNP models are capable of predicting a real system's detector responses when the models are constructed as realistically as possible. 

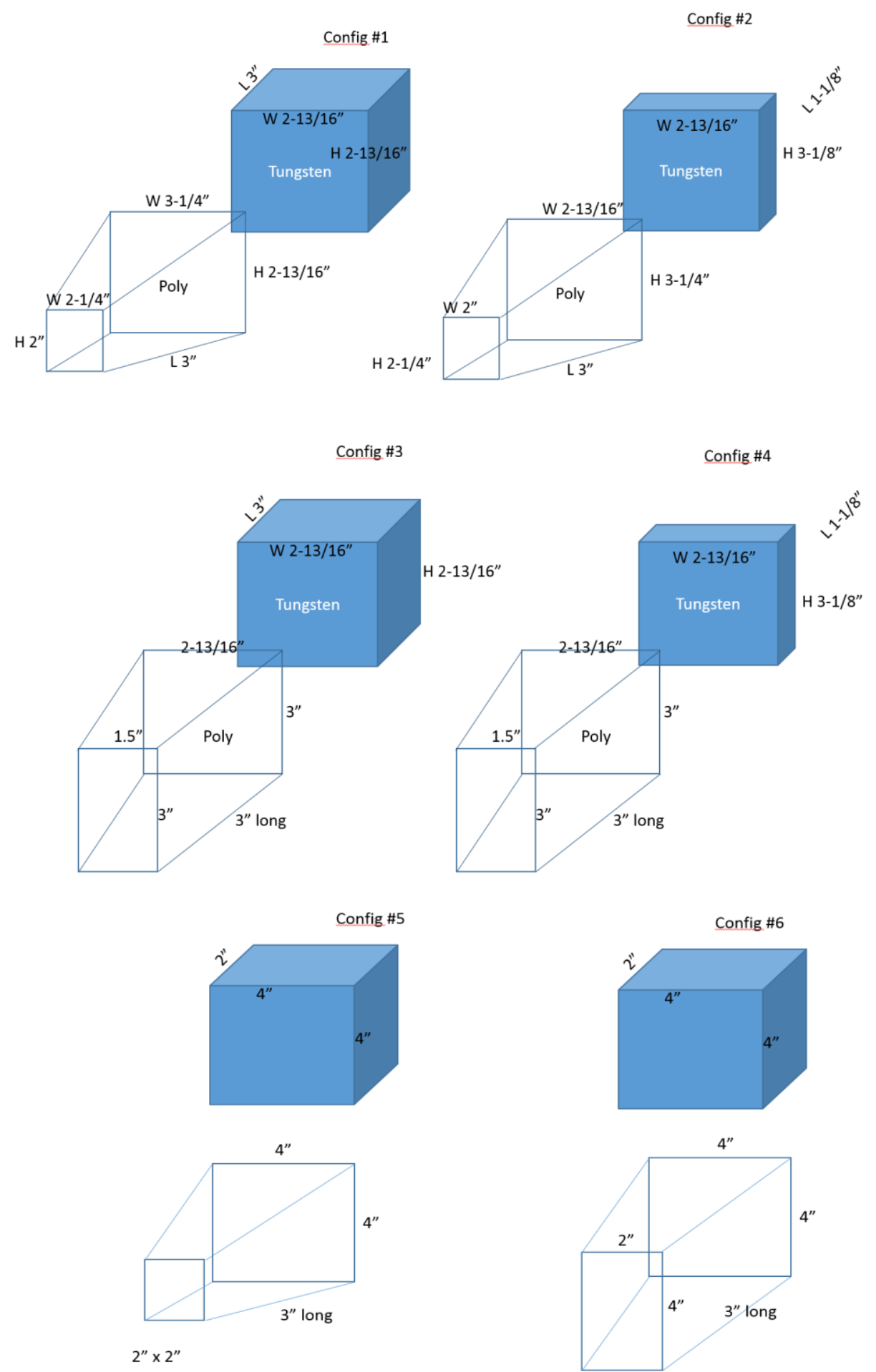

Figure 1. Six configurations of the shadow shield used in the experiments by combining tungsten and HDPE blocks (not drawn to scale). 
Table 1. Summary of experimental data analyzed in this study. Configuration numbers correspond to those assigned in Figure 1. Live, real and dead time are in seconds. The last column is spectral quality calculated in PINS+ analysis. The P385 D-D generator was operated at $130 \mathrm{kV}$ and $70 \mu \mathrm{A}$.

\begin{tabular}{|c|c|c|c|c|c|c|c|}
\hline Configuration \# & Run \# & simulant & File name & Live time & Real time & Dead time & Spec. Q. \\
\hline 4 & 1 & $\mathrm{HD}$ & P09_220ct18_003 & 3000 & 4529.04 & $33.76 \%$ & 89.65 \\
\hline 6 & 1 & HD & P09_250ct18_150 & 3000 & 3360.18 & $10.72 \%$ & 100.00 \\
\hline 3 & 1 & $H D$ & P09_180ct18_030 & 3000 & 3776.30 & $20.56 \%$ & 96.82 \\
\hline 2 & 1 & HD & P09_100ct18_030 & 3000 & 4158.18 & $27.85 \%$ & 100.00 \\
\hline 5 & 1 & $\mathrm{HD}$ & P09_240ct18_030 & 3000 & 3415.50 & $12.17 \%$ & 91.89 \\
\hline 1 & 1 & $\mathrm{HD}$ & P09_150ct18_030 & 3000 & 3545.36 & $15.38 \%$ & 100.00 \\
\hline 4 & 2 & $\mathrm{HD}$ & P09_220ct18_015 & 3000 & 4317.16 & $30.51 \%$ & 100.00 \\
\hline 6 & 2 & $\mathrm{HD}$ & P09_290ct18_120 & 3000 & 3379.72 & $11.24 \%$ & 95.37 \\
\hline 3 & 2 & HD & P09_180ct18_150 & 3000 & 3892.02 & $22.92 \%$ & 100.00 \\
\hline 2 & 2 & $\mathrm{HD}$ & P09_100ct18_150 & 3000 & 4404.98 & $31.90 \%$ & 100.00 \\
\hline 5 & 2 & $\mathrm{HD}$ & P09_240ct18_150 & 3000 & 3443.70 & $12.88 \%$ & 100.00 \\
\hline 1 & 2 & $\mathrm{HD}$ & P09_150ct18_150 & 3000 & 3537.98 & $15.21 \%$ & 100.00 \\
\hline 4 & 3 & $\mathrm{HD}$ & P09_230ct18_009 & 3000 & 4298.64 & $30.21 \%$ & 96.15 \\
\hline 6 & 3 & HD & P09_290ct18_240 & 3000 & 3394.68 & $11.63 \%$ & 94.73 \\
\hline 3 & 3 & HD & P09_180ct18_270 & 3000 & 3995.70 & $24.92 \%$ & 100.00 \\
\hline 2 & 3 & $\mathrm{HD}$ & P09_110ct18_090 & 3000 & 4222.16 & $28.95 \%$ & 93.68 \\
\hline 5 & 3 & $\mathrm{HD}$ & P09_250ct18_030 & 3000 & 3387.02 & $11.43 \%$ & 92.59 \\
\hline 1 & 3 & $\mathrm{HD}$ & P09_170ct18_090 & 3000 & 3771.10 & $20.45 \%$ & 93.84 \\
\hline 4 & 1 & VX & P09_220ct18_012 & 3000 & 4389.60 & $31.66 \%$ & 97.03 \\
\hline 6 & 1 & VX & P09_290ct18_090 & 3000 & 3396.04 & $11.66 \%$ & 90.42 \\
\hline 3 & 1 & VX & P09_180ct18_120 & 3000 & 3904.26 & $23.16 \%$ & 100.00 \\
\hline 2 & 1 & VX & P09_100ct18_120 & 3000 & 4133.04 & $27.41 \%$ & 96.31 \\
\hline 5 & 1 & VX & P09_240ct18_120 & 3000 & 3435.86 & $12.69 \%$ & 98.44 \\
\hline 1 & 1 & VX & P09_15Oct18_120 & 3000 & 3538.18 & $15.21 \%$ & 99.07 \\
\hline 4 & 2 & VX & P09_230ct18_006 & 3000 & 4308.52 & $30.37 \%$ & 93.30 \\
\hline 6 & 2 & VX & P09_290ct18_210 & 3000 & 3405.34 & $11.90 \%$ & 94.44 \\
\hline 3 & 2 & VX & P09_180ct18_240 & 3000 & 3999.02 & $24.98 \%$ & 100.00 \\
\hline 2 & 2 & VX & P09_110ct18_060 & 3000 & 4270.72 & $29.75 \%$ & 100.00 \\
\hline 5 & 2 & VX & P09_240ct18_240 & 3000 & 3421.94 & $12.33 \%$ & 97.56 \\
\hline 1 & 2 & VX & P09_170ct18_060 & 3000 & 3804.72 & $21.15 \%$ & 99.52 \\
\hline 4 & 3 & VX & P09_230ct18_018 & 3000 & 4043.02 & $25.80 \%$ & 96.03 \\
\hline 6 & 3 & $\mathrm{VX}$ & P09_300ct18_060 & 3000 & 3418.56 & $12.24 \%$ & 86.79 \\
\hline 3 & 3 & VX & P09_190ct18_090 & 3000 & 3856.36 & $22.21 \%$ & 97.60 \\
\hline 2 & 3 & $\mathrm{VX}$ & P09_110ct18_180 & 3000 & 4153.26 & $27.77 \%$ & 96.10 \\
\hline 5 & 3 & VX & P09_250ct18_120 & 3000 & 3391.80 & $11.55 \%$ & 92.00 \\
\hline 1 & 3 & $\mathrm{VX}$ & P09_170ct18_180 & 3000 & 3698.84 & $18.89 \%$ & 97.05 \\
\hline
\end{tabular}


Table 1. Continued.

\begin{tabular}{|c|c|c|c|c|c|c|c|}
\hline Configuration \# & Repeat \# & Simulant & File name & Live time & Real time & Dead time & Spec. Q. \\
\hline 4 & 1 & GB & P09_220ct18_009 & 3000 & 4595.66 & $34.72 \%$ & 95.68 \\
\hline 6 & 1 & GB & P09_290ct18_060 & 3000 & 3398.24 & $11.72 \%$ & 90.57 \\
\hline 3 & 1 & GB & P09_180ct18_090 & 3000 & 3871.48 & $22.51 \%$ & 99.49 \\
\hline 2 & 1 & GB & P09_100ct18_090 & 3000 & 4172.14 & $28.09 \%$ & 96.04 \\
\hline 5 & 1 & GB & P09_240ct18_090 & 3000 & 3432.8 & $12.61 \%$ & 94.92 \\
\hline 1 & 1 & GB & P09_150ct18_090 & 3000 & 3543.82 & $15.35 \%$ & 93.83 \\
\hline 4 & 2 & GB & P09_230ct18_003 & 3000 & 4292.40 & $30.11 \%$ & 90.44 \\
\hline 6 & 2 & GB & P09_290ct18_180 & 3000 & 3403.08 & $11.84 \%$ & 97.02 \\
\hline 3 & 2 & GB & P09_180ct18_210 & 3000 & 4045.28 & $25.84 \%$ & 100.00 \\
\hline 2 & 2 & GB & P09_110ct18_030 & 3000 & 4694.90 & $36.10 \%$ & 95.01 \\
\hline 5 & 2 & GB & P09_240ct18_210 & 3000 & 3432.06 & $12.59 \%$ & 95.60 \\
\hline 1 & 2 & GB & P09_170ct18_030 & 3000 & 3671.10 & $18.28 \%$ & 94.24 \\
\hline 4 & 3 & GB & P09_230ct18_015 & 3000 & 4073.56 & $26.35 \%$ & 96.78 \\
\hline 6 & 3 & GB & P09_300ct18_030 & 3000 & 3425.66 & $12.43 \%$ & 87.95 \\
\hline 3 & 3 & GB & P09_190ct18_060 & 3000 & 3921.98 & $23.51 \%$ & 94.62 \\
\hline 2 & 3 & GB & P09_110ct18_150 & 3000 & 4191.78 & $28.43 \%$ & 96.33 \\
\hline 5 & 3 & GB & P09_250ct18_090 & 3000 & 3506.24 & $14.44 \%$ & 88.12 \\
\hline 1 & 3 & GB & P09_170ct18_150 & 3000 & 3776.08 & $20.55 \%$ & 95.21 \\
\hline 4 & 1 & L & P09_220ct18_006 & 3000 & 4580.30 & $34.50 \%$ & 97.61 \\
\hline 6 & 1 & L & P09_290ct18_030 & 3000 & 3374.24 & $11.09 \%$ & 86.84 \\
\hline 3 & 1 & L & P09_180ct18_060 & 3000 & 3852.06 & $22.12 \%$ & 96.66 \\
\hline 2 & 1 & $\mathrm{~L}$ & P09_100ct18_060 & 3000 & 4162.36 & $27.93 \%$ & 100.00 \\
\hline 5 & 1 & L & P09_240ct18_060 & 3000 & 3421.76 & $12.33 \%$ & 96.92 \\
\hline 1 & 1 & L & P09_150ct18_060 & 3000 & 3541.14 & $15.28 \%$ & 100.00 \\
\hline 4 & 2 & L & P09_220ct18_018 & 3000 & 4240.84 & $29.26 \%$ & 95.50 \\
\hline 6 & 2 & L & P09_290ct18_150 & 3000 & 3389.96 & $11.50 \%$ & 93.87 \\
\hline 3 & 2 & $\mathrm{~L}$ & P09_180ct18_180 & 3000 & 4016.82 & $25.31 \%$ & 100.00 \\
\hline 2 & 2 & L & P09_100ct18_180 & 3000 & 4923.26 & $39.06 \%$ & 97.64 \\
\hline 5 & 2 & L & P09_240ct18_180 & 3000 & 3438.46 & $12.75 \%$ & 100.00 \\
\hline 1 & 2 & L & P09_150ct18_180 & 3000 & 3567.68 & $15.91 \%$ & 100.00 \\
\hline 4 & 3 & L & P09_230ct18_012 & 3000 & 4150.84 & $27.73 \%$ & 95.40 \\
\hline 6 & 3 & $\mathrm{~L}$ & P09_290ct18_270 & 3000 & 3421.88 & $12.33 \%$ & 96.51 \\
\hline 3 & 3 & $\mathrm{~L}$ & P09_190ct18_030 & 3000 & 3943.62 & $23.93 \%$ & 97.61 \\
\hline 2 & 3 & $\mathrm{~L}$ & P09_110ct18_120 & 3000 & 4228.16 & $29.05 \%$ & 96.93 \\
\hline 5 & 3 & $\mathrm{~L}$ & P09_250ct18_060 & 3000 & 3396.36 & $11.67 \%$ & 93.82 \\
\hline 1 & 3 & L & P09_170ct18_120 & 3000 & 3787.14 & $20.78 \%$ & 100.00 \\
\hline
\end{tabular}



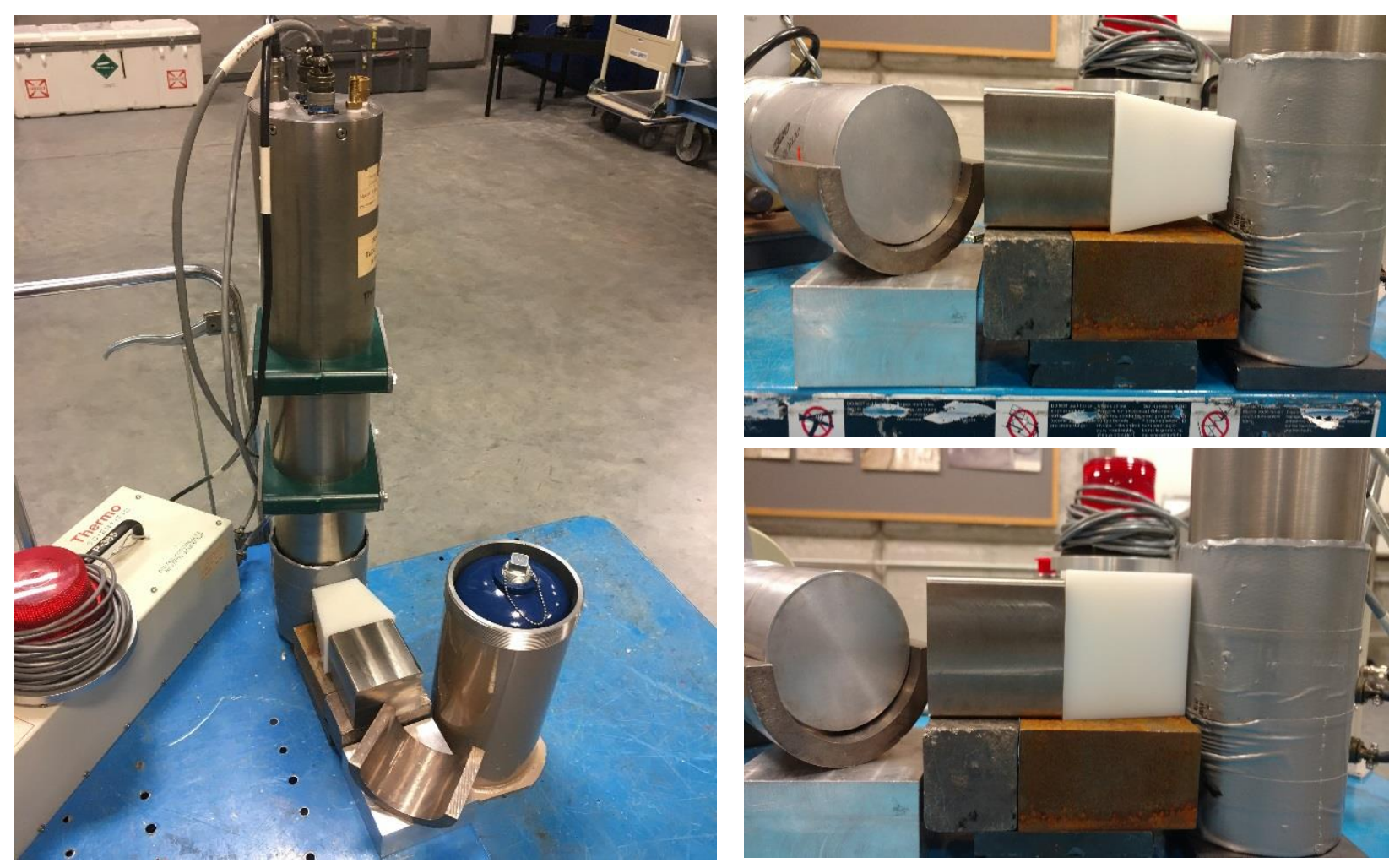

Figure 2. (Left) PINS laboratory setup with a P385 D-D generator and a simulant in place. Shadow shield configuration \#1 (top right) and (bottom right) \#3. Iron and aluminum blocks were used to align centers of the detector, shadow shield and the generator target line at the same height. These improvised metal blocks and the concrete floor were also included in MCNP models, but the generator control module and the blue cart were not modeled.
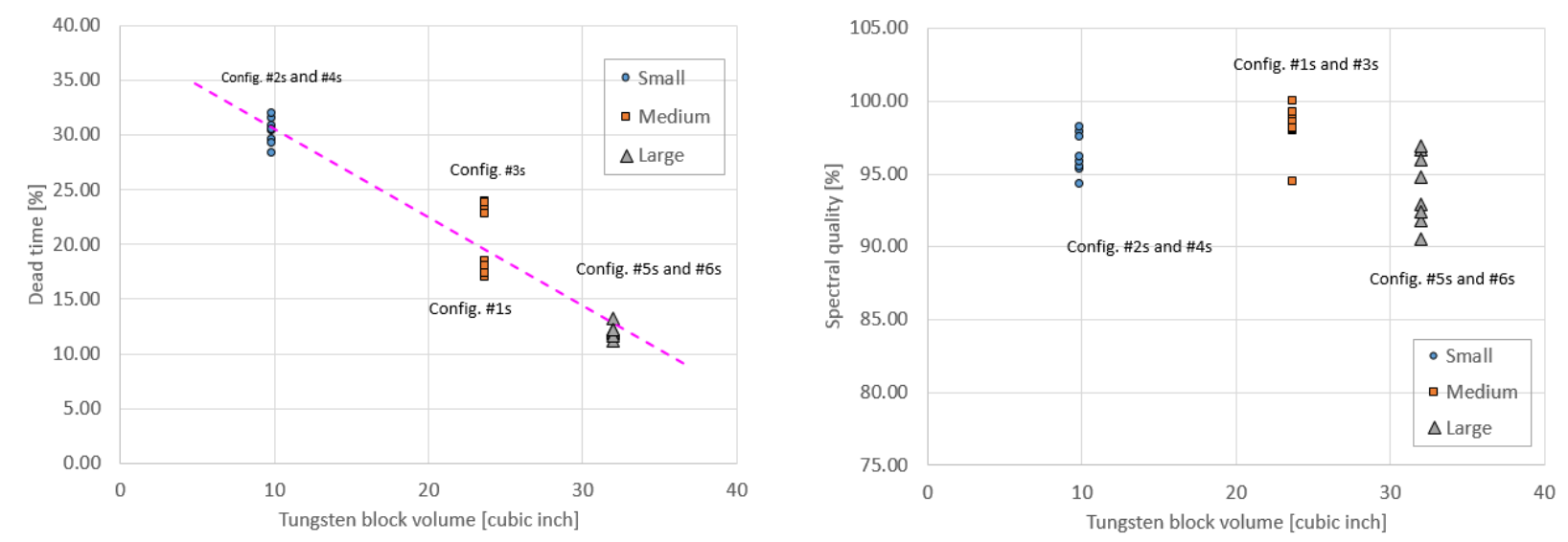

Figure 3. (Left) averaged dead times of the measured spectra are plotted as a function of the tungsten block volume. (Right) averaged spectral qualities of the measured spectra are plotted as a function of the tungsten block volume. Data points are grouped together according to the tungsten block volumes defined as small (configuration \#2 and \#4), medium (configuration \#1 and \#3) and large (configuration \#5 and \#6). 


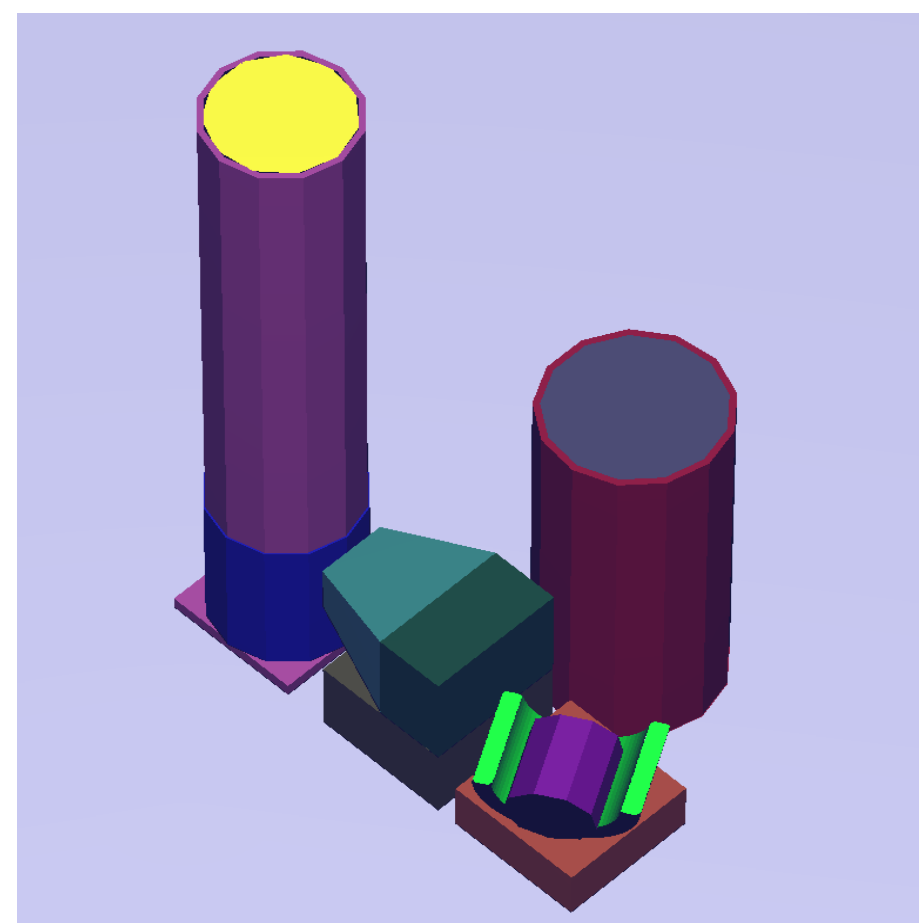

Figure 4. MCNP model of laboratory setup with the shadow shield configuration \#5 in place. MCNP models were built to describe as close to the real setups as possible. As discussed above, the generator control module and the blue cart were not modeled under the assumption that those have negligible impact on the simulation results.
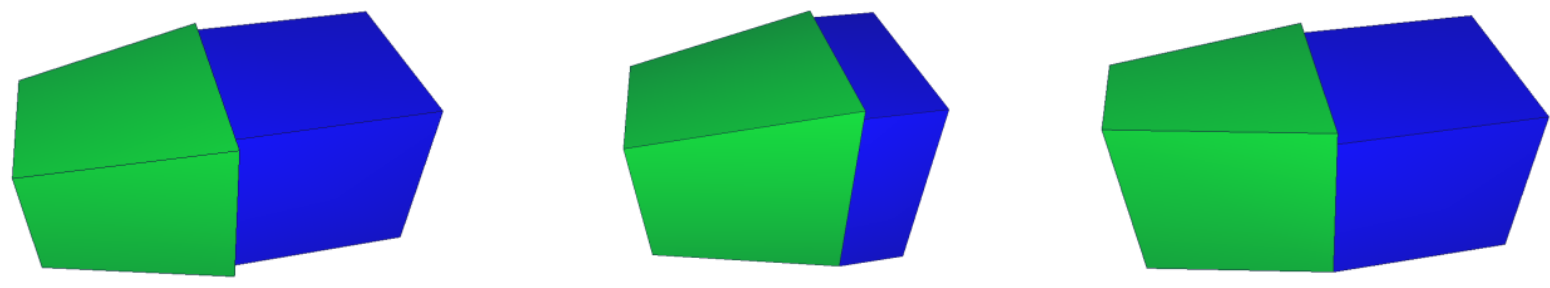

Configuration \#1 (pyramid HDPE + medium W)

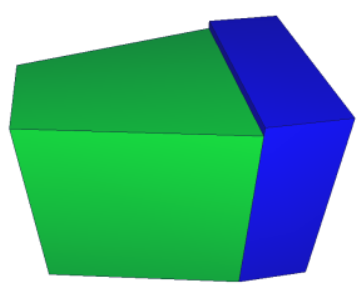

Configuration \#4 (wedge HDPE + small W)
Configuration \#2 (pyramid HDPE + small W)

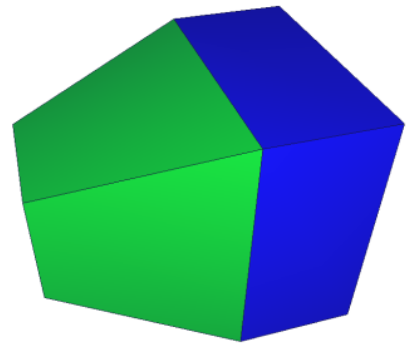

Configuration \#5 (pyramid HDPE + large W)
Configuration \#3 (wedge HDPE + medium W)

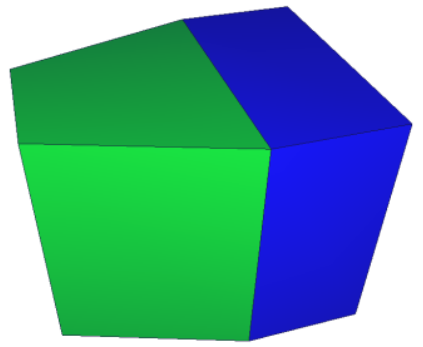

Configuration \#6 (wedge HDPE + large W)

Figure 5. MCNP models of six unique shadow shield configurations. Actual dimensions of the HDPE (green) and tungsten (blue) blocks were used to reproduce these shadow shield in MCNP input decks. 
Table 2. Measured and simulated net counts of hydrogen in background spectra. Two independent background spectra were collected for each configuration. Average value of those two was adopted for each configuration's experimental value in this table. All MCNP results were multiplied by $6.56 \times 10^{9}$ as discussed in section 5.1

\begin{tabular}{|c|c|c|c|}
\hline \multirow{2}{*}{ Configuration \# } & \multirow{2}{*}{ Simulant } & Experiment & MCNP \\
\hline & & 2223keV & $2223 \mathrm{keV}$ \\
\hline 4 (wedge + small W) & Background & 1093 & 1093 \\
\hline 6 (wedge + large W) & Background & 699 & 740 \\
\hline 3 (wedge + medium W) & Background & 337 & 424 \\
\hline $2($ pyramid + small W) & Background & 1204 & 1158 \\
\hline 5 (pyramid + large W) & Background & 534 & 651 \\
\hline 1 (pyramid + medium $W)$ & Background & 349 & 445 \\
\hline
\end{tabular}

\section{H 2223keV}

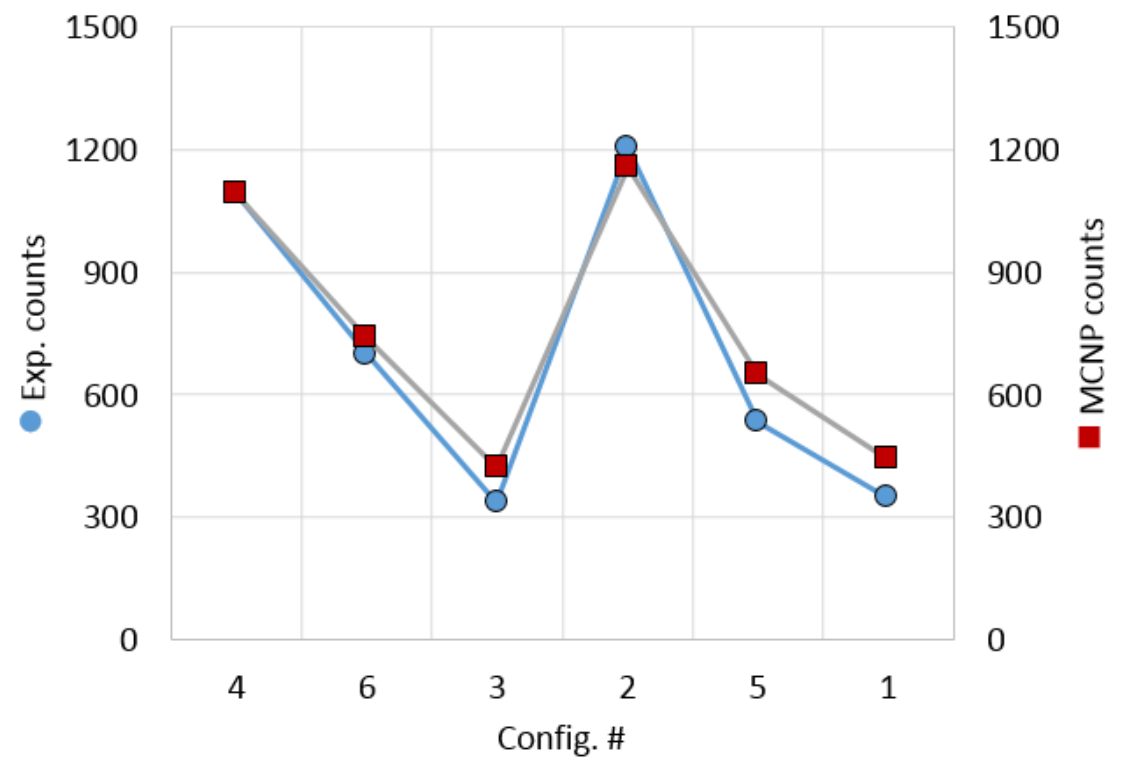

Figure 6. Net counts of $2223 \mathrm{keV}$ in background spectra. $2223 \mathrm{keV}$ gamma-rays from hydrogen occur in HDPE block in the shadow shield. Solid circle symbols represent experimental data and cross symbols represent MCNP results. 

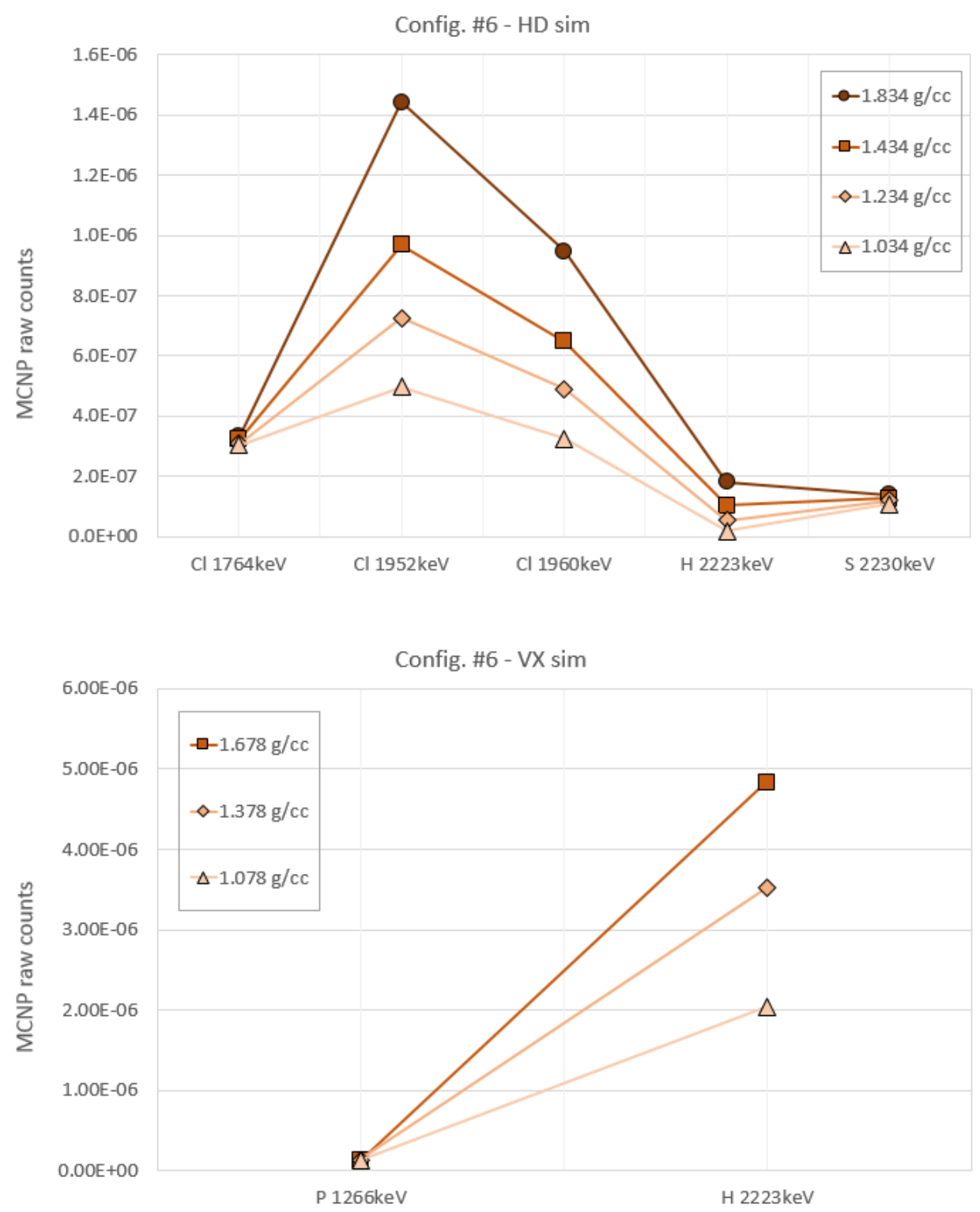

Figure 7. (Top) Changes in net counts for various HD simulant densities. Density of The HD simulant was varied from 1.034 to $1.834 \mathrm{~g} / \mathrm{cm}^{3}$ in increments of $0.200 \mathrm{~g} / \mathrm{cm}^{3}$. (Bottom) Changes in net counts for various VX simulant densities. Density of the VX simulant was varied from 1.078 to $1.678 \mathrm{~g} / \mathrm{cm}^{3}$ in increments of $0.300 \mathrm{~g} / \mathrm{cm}^{3}$. Only configuration \#6 was tested in this study, but the same detector responses are expected to be observed regardless of configurations. 
Table 3. Measure and simulated net counts of chlorine, hydrogen and sulfur for The HD simulant. Three independent spectra were collected for each configuration. Average value of those three was adopted for each configuration's experimental value in this table. The HD simulant's density of $1.434 \mathrm{~g} / \mathrm{cm}^{3}$ was used in MCNP simulations. All MCNP results were multiplied by $5.43 \times 10^{9}$ as discussed in section 5.2.

\begin{tabular}{|c|c|c|c|c|c|c|c|c|c|c|c|}
\hline \multirow{2}{*}{ Configuration \# } & \multirow{2}{*}{ Simulant } & \multicolumn{5}{|c|}{ Experiment } & \multicolumn{5}{|c|}{ MCNP } \\
\hline & & $1764 \mathrm{keV}$ & $1952 \mathrm{keV}$ & 1960keV & $2223 \mathrm{keV}$ & $2230 \mathrm{keV}$ & 1764keV & $1952 \mathrm{keV}$ & $1960 \mathrm{keV}$ & $2223 \mathrm{keV}$ & $2230 \mathrm{keV}$ \\
\hline 4 & $\mathrm{HD}$ & 2171 & 2339 & 1475 & 401 & 371 & 2553 & 6573 & 4234 & 1073 & 934 \\
\hline 6 & $\mathrm{HD}$ & 1997 & 2563 & 1688 & 478 & 382 & 1758 & 5256 & 3522 & 570 & 687 \\
\hline 3 & $\mathrm{HD}$ & 1699 & 1713 & 1265 & 141 & 337 & 1724 & 4452 & 2968 & 143 & 647 \\
\hline 2 & $\mathrm{HD}$ & 2683 & 3000 & 1976 & 245 & 592 & 2520 & 6566 & 4210 & 1120 & 929 \\
\hline 5 & $\mathrm{HD}$ & 1799 & 2168 & 1443 & 390 & 396 & 1796 & 5136 & 3433 & 491 & 713 \\
\hline 1 & $\mathrm{HD}$ & 1943 & 2061 & 1294 & 169 & 454 & 1430 & 4192 & 2724 & 105 & 552 \\
\hline
\end{tabular}

$\mathrm{Cl} 1764 \mathrm{keV}$

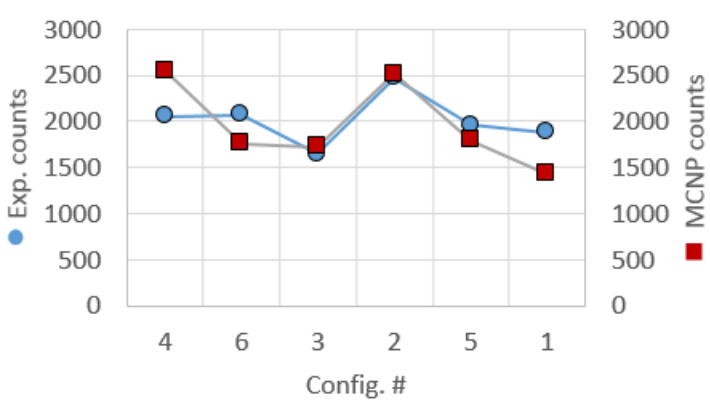

Cl 1960keV

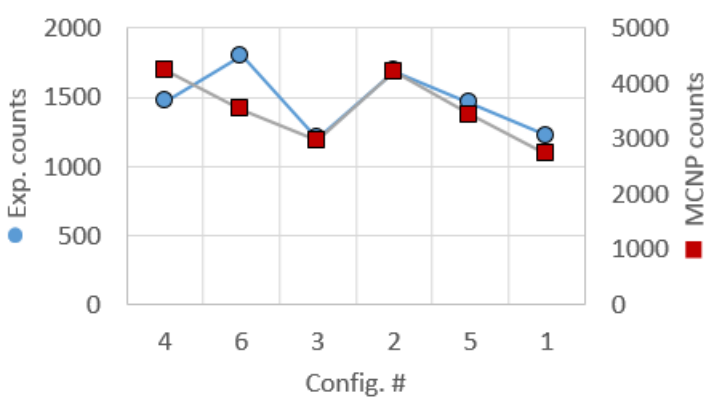

S $2230 \mathrm{keV}$

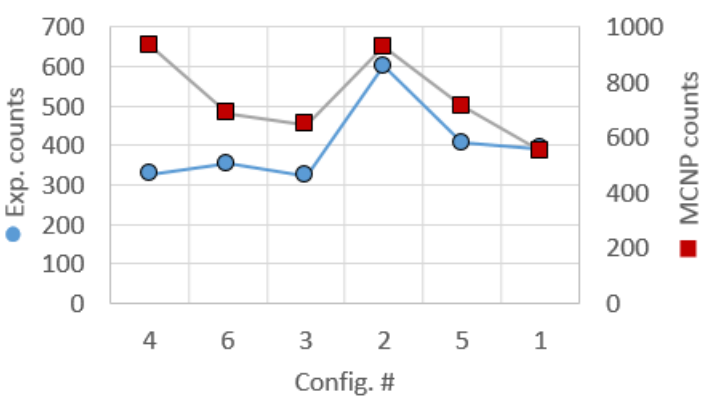

Cl 1952keV

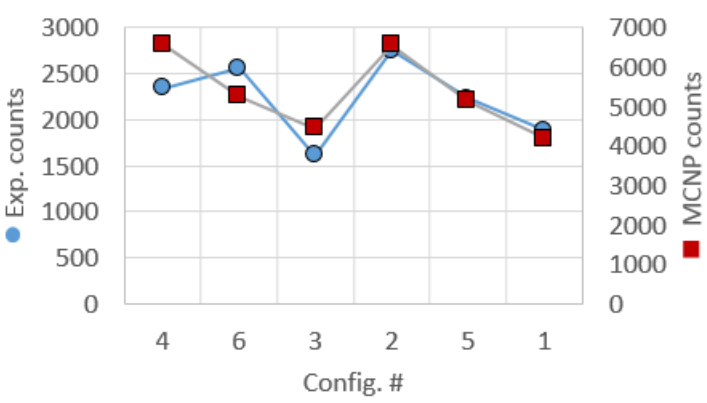

H $2223 \mathrm{keV}$

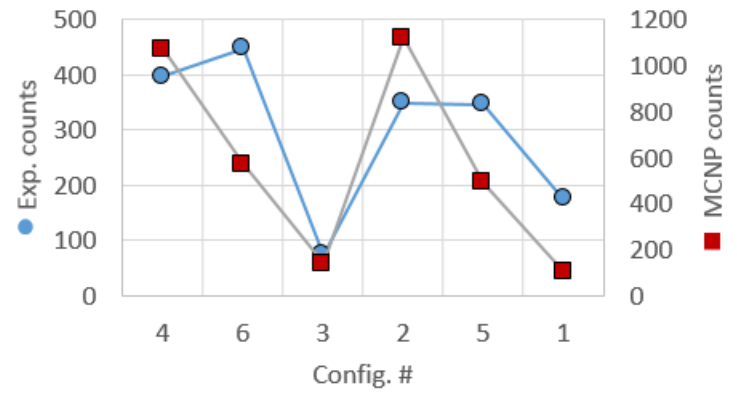

Figure 8. Net counts of gamma-ray peaks from key elements in The HD simulant: 1764, 1952 and $1960 \mathrm{keV}$ from chlorine, $2223 \mathrm{keV}$ from hydrogen and $2230 \mathrm{keV}$ from sulfur. Solid circle symbols represent experimental data and square symbols represent MCNP results, assuming The HD simulant's density is $1.434 \mathrm{~g} / \mathrm{cm}^{3}$. 
Cl $1764 \mathrm{keV}$

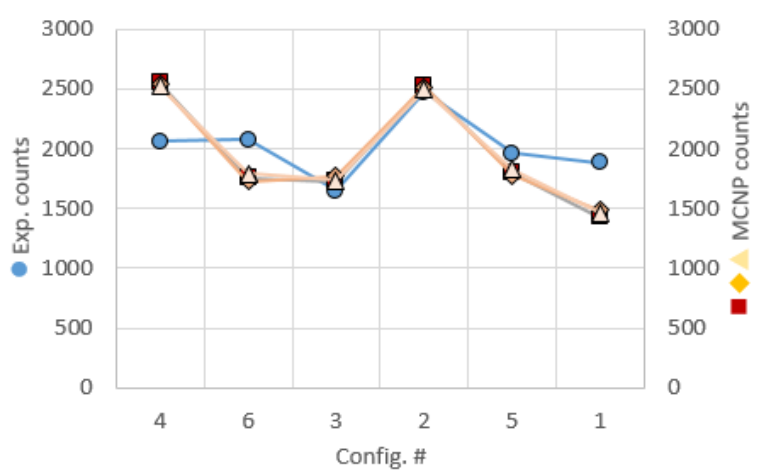

Cl 1960keV

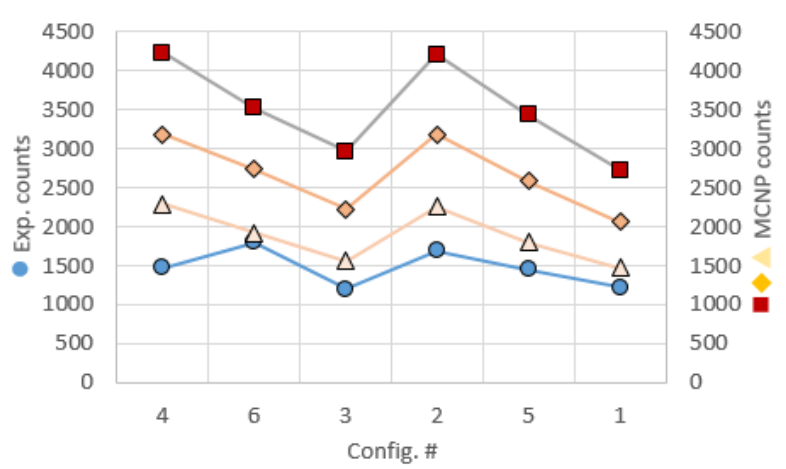

H $2223 \mathrm{eV}$

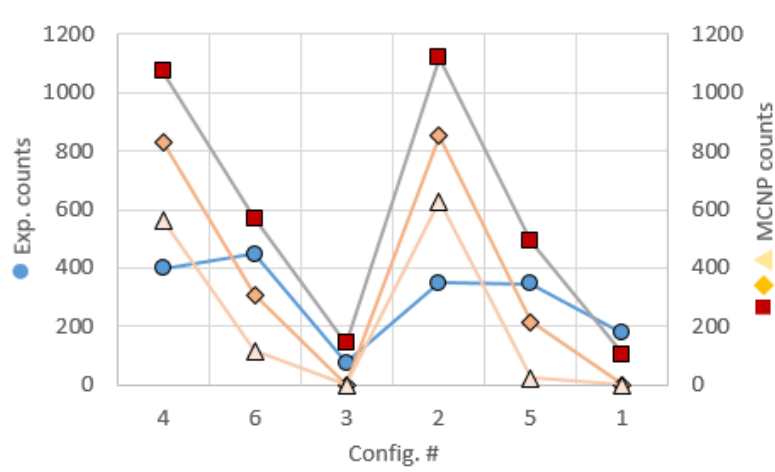

$\mathrm{Cl} 1952 \mathrm{eV}$

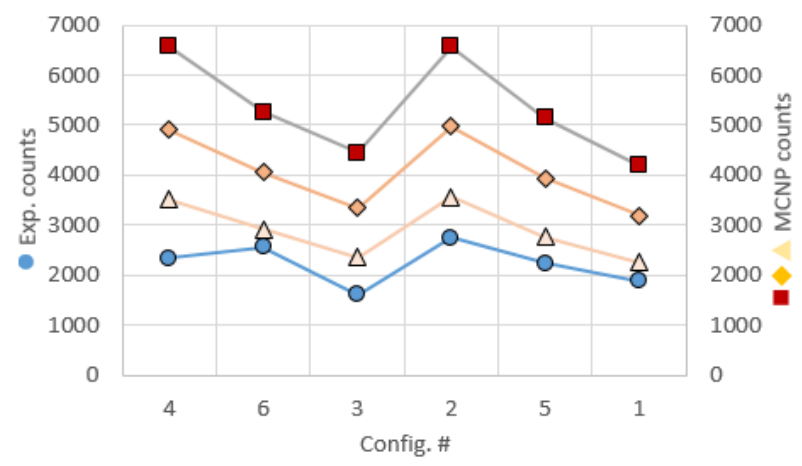

S 2230keV
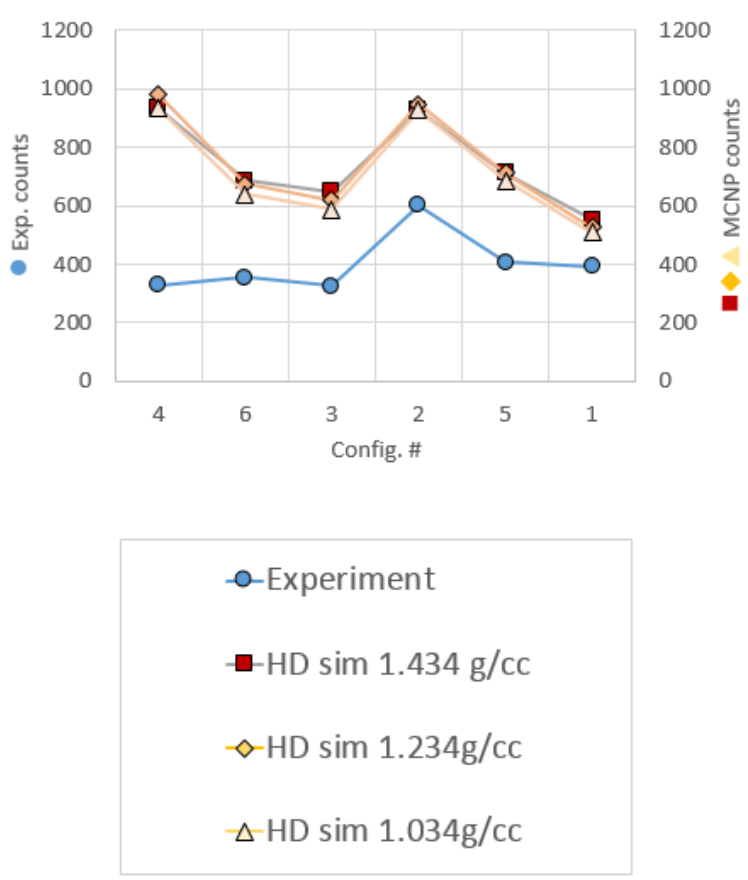

Figure 9. Net counts of gamma-ray peaks from key elements in The HD simulant: 1764, 1952 and $1960 \mathrm{keV}$ from chlorine, $2223 \mathrm{keV}$ from hydrogen and $2230 \mathrm{keV}$ from sulfur. Solid circle symbols ( $\bullet$ ) represent experimental data and square symbols (ם) represent MCNP results, assuming The HD simulant's density is $1.434 \mathrm{~g} / \mathrm{cm}^{3}$. Solid diamond $(\diamond)$ symbols and solid triangle $(\triangle)$ symbols represent MCNP results, assuming The HD simulant's densities are $1.234 \mathrm{~g} / \mathrm{cm}^{3}$ and $1.034 \mathrm{~g} / \mathrm{cm}^{3}$, respectively. 
Table 4. Measured and simulated net counts of phosphorus and hydrogen for the VX simulant. Three independent spectra were collected for each configuration. Average value of those three was adopted for each configuration's experimental value in this table. The VX simulant's density of $1.378 \mathrm{~g} / \mathrm{cm}^{3}$ was used in MCNP simulations. All MCNP results were multiplied by $9.68 \times 10^{9}$ as discussed in section 5.3.

\begin{tabular}{|c|c|c|c|c|c|}
\hline \multirow{2}{*}{ Configuration \# } & \multirow{2}{*}{ Simulant } & \multicolumn{2}{|c|}{ Experiment } & \multicolumn{2}{|c|}{ MCNP } \\
\hline & & $1266 \mathrm{keV}$ & $2223 \mathrm{keV}$ & $1266 \mathrm{keV}$ & $2223 \mathrm{keV}$ \\
\hline 4 & VX & 1893 & 18405 & 2552 & 43290 \\
\hline 6 & $\mathrm{VX}$ & 1881 & 19656 & 1447 & 34190 \\
\hline 3 & VX & 1650 & 12762 & 1358 & 29043 \\
\hline 2 & $\mathrm{VX}$ & 2332 & 19043 & 2441 & 43263 \\
\hline 5 & $\mathrm{VX}$ & 1857 & 18407 & 1591 & 34048 \\
\hline 1 & $\mathrm{VX}$ & 1533 & 14160 & 1059 & 26732 \\
\hline
\end{tabular}

P 1266keV

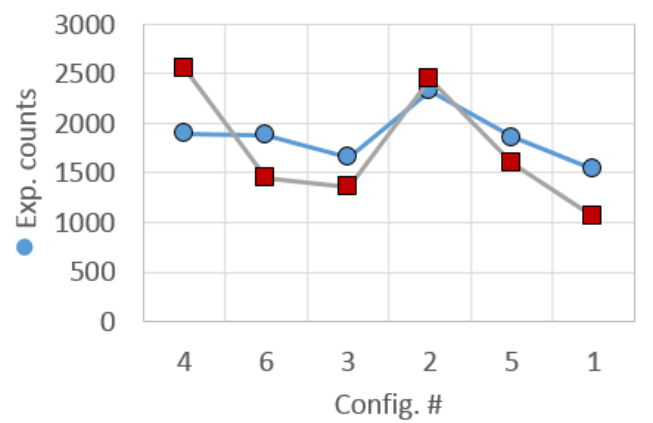

H $2223 \mathrm{keV}$

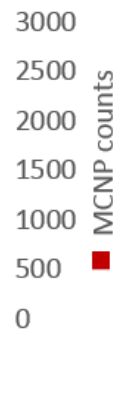

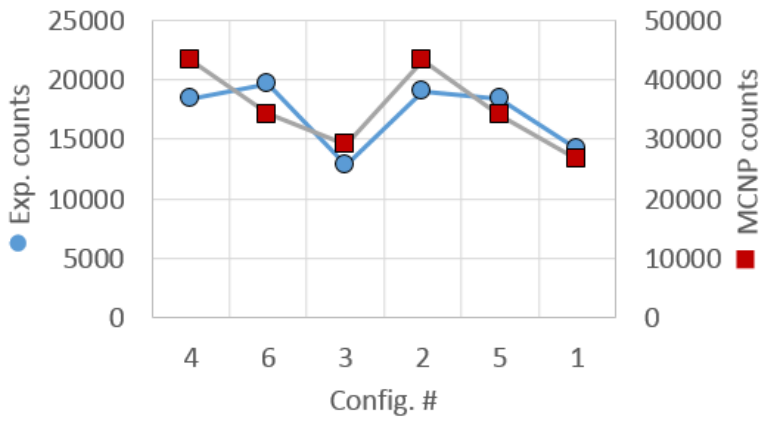

Figure 10. Net counts of gamma-ray peaks from key elements in the VX simulant: 1266keV from phosphorus and $2223 \mathrm{keV}$ from hydrogen. Solid circle symbols represent experimental data and square symbols represent MCNP results, assuming the VX simulant's density is $1.378 \mathrm{~g} / \mathrm{cm}^{3}$. 
Table 5. Measured and simulated net counts of phosphorus and hydrogen for the GB simulant. Three independent spectra were collected for each configuration. Average value of those three was adopted for each configuration's experimental value in this table. The GB simulant's density of $1.820 \mathrm{~g} / \mathrm{cm}^{3}$ was used in MCNP simulations. All MCNP results were multiplied by $6.04 \times 10^{9}$ as discussed in section 5.4.

\begin{tabular}{|c|c|c|c|c|c|}
\hline \multirow{2}{*}{ Configuration \# } & \multirow{2}{*}{ Simulant } & \multicolumn{2}{|c|}{ Experiment } & \multicolumn{3}{c|}{ MCNP } \\
\cline { 3 - 6 } & & $1266 \mathrm{keV}$ & $2223 \mathrm{keV}$ & $1266 \mathrm{keV}$ & 4833 \\
\hline 4 & GB & 3976 & 14798 & $2223 \mathrm{keV}$ \\
\hline 6 & GB & 3621 & 16180 & 2899 & 19556 \\
\hline 3 & GB & 3110 & 10856 & 4673 & 12935 \\
\hline 2 & GB & 4267 & 15771 & 3112 & 15429 \\
\hline 5 & GB & 3476 & 12523 & 2276 & 11958 \\
\hline 1 & GB & 3270 & & & \\
\hline
\end{tabular}

P 1266keV

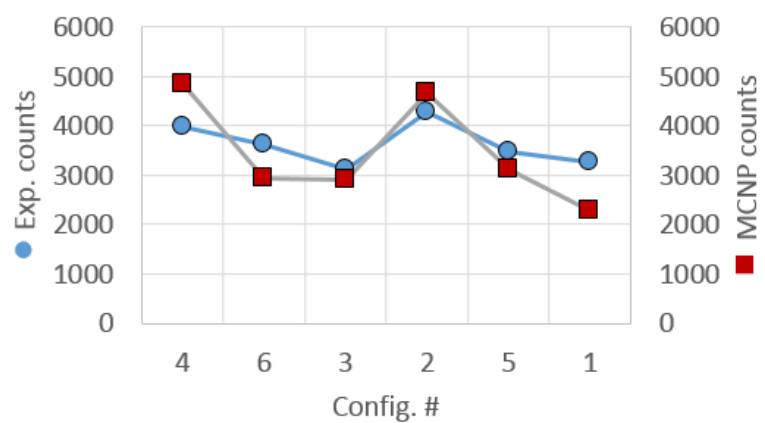

H 2223keV

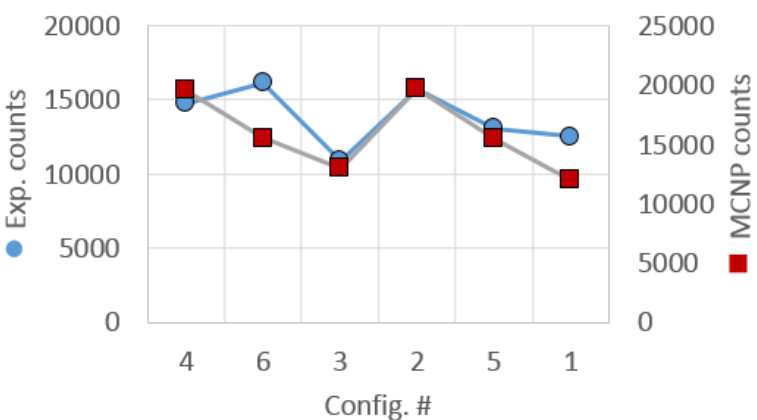

Figure 11. Net counts of gamma-ray peaks from key elements in the GB simulant: $12662 \mathrm{keV}$ from phosphorus and $2223 \mathrm{keV}$ from hydrogen. Solid circle symbols represent experimental data and square symbols represent MCNP results, assuming the GB simulant's density is $1.820 \mathrm{~g} / \mathrm{cm}^{3}$. 
Table 6. Measured and simulated net counts of chlorine, hydrogen and sulfur for the L simulant. Three independent spectra were collected for each configuration. Average value of those three was adopted for each configuration's experimental value in this table. The L simulant's density of $1.895 \mathrm{~g} / \mathrm{cm}^{3}$ was used in MCNP simulations. All MCNP results were multiplied by $4.49 \times 10^{9}$ as discussed in section 5.5.

\begin{tabular}{|c|c|c|c|c|c|c|c|c|c|c|c|}
\hline \multirow{2}{*}{ Configuration \# } & \multirow{2}{*}{ Simulant } & \multicolumn{5}{|c|}{ Experiment } & \multicolumn{5}{|c|}{ MCNP } \\
\hline & & $1764 \mathrm{keV}$ & $1952 \mathrm{keV}$ & 1960keV & 2223keV & $279 \mathrm{keV}$ & $1764 \mathrm{keV}$ & $1952 \mathrm{keV}$ & 1960keV & $2223 \mathrm{keV}$ & $279 \mathrm{keV}$ \\
\hline 4 & $\mathrm{~L}$ & 3470 & 1109 & 658 & 49 & 20467 & 4079 & 631 & 383 & 15 & 12308 \\
\hline 6 & $\mathrm{~L}$ & 3245 & 988 & 625 & 1 & 19160 & 2777 & 552 & 323 & 0 & 8073 \\
\hline 3 & $\mathrm{~L}$ & 2785 & 481 & 389 & 0 & 16407 & 2736 & 373 & 217 & 0 & 7333 \\
\hline 2 & $\mathrm{~L}$ & 4235 & 1088 & 784 & 0 & 24953 & 4023 & 669 & 396 & 61 & 12009 \\
\hline 5 & $\mathrm{~L}$ & 2974 & 906 & 535 & 100 & 17635 & 2777 & 552 & 323 & 0 & 8073 \\
\hline 1 & $\mathrm{~L}$ & 2834 & 702 & 376 & 0 & 16410 & 2288 & 389 & 251 & 0 & 6481 \\
\hline
\end{tabular}

$\mathrm{Cl} 1764 \mathrm{keV}$

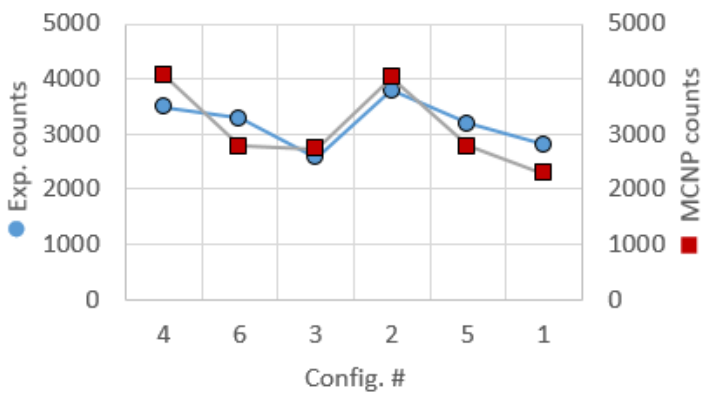

Cl 1960keV

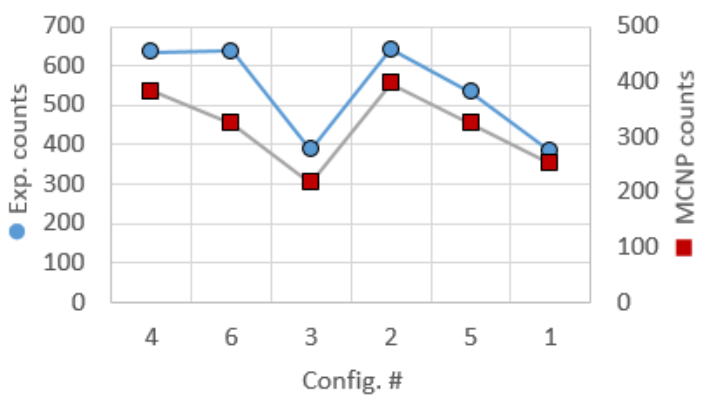

As $279 \mathrm{keV}$

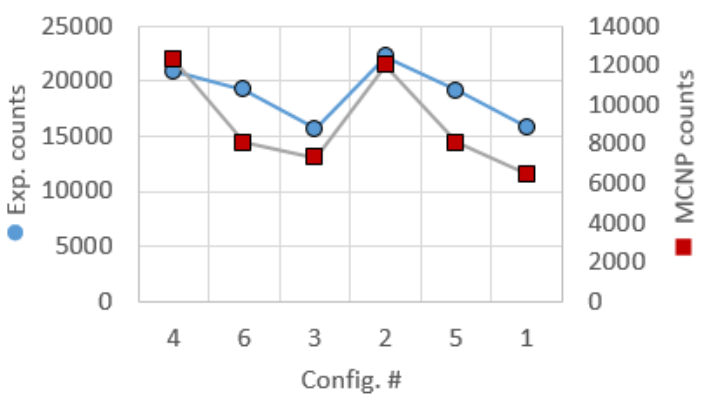

Cl $1952 \mathrm{keV}$

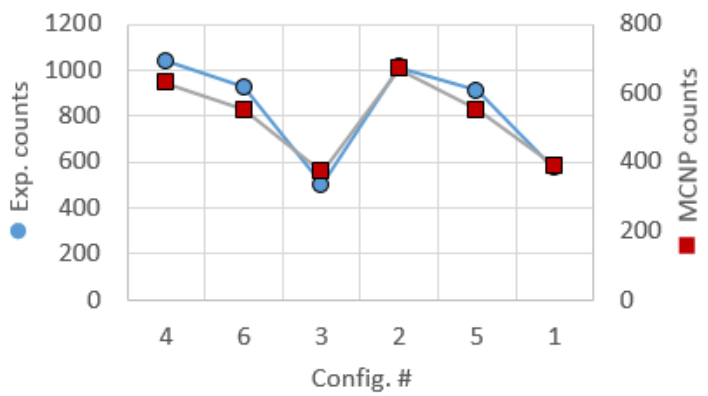

H 2223keV

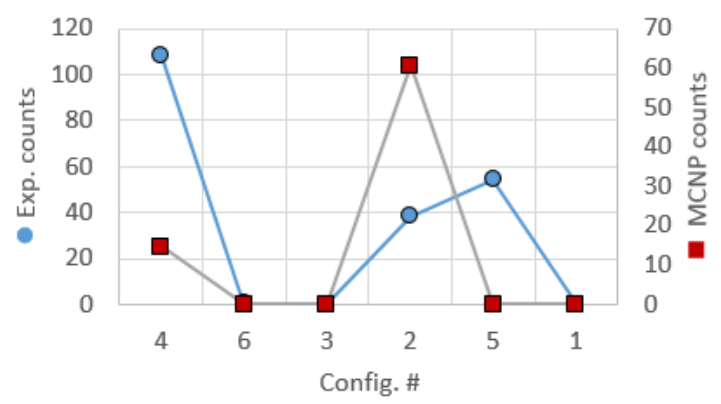

Figure 12. Net counts of gamma-ray peaks from key elements in the L simulant: 1764, 1952 and $1960 \mathrm{keV}$ from chlorine, $2223 \mathrm{keV}$ from hydrogen and $279 \mathrm{keV}$ from arsenic. Solid circle symbols represent experimental data and square symbols represent MCNP results, assuming the L simulant's density is $1.895 \mathrm{~g} / \mathrm{cm}^{3}$. 


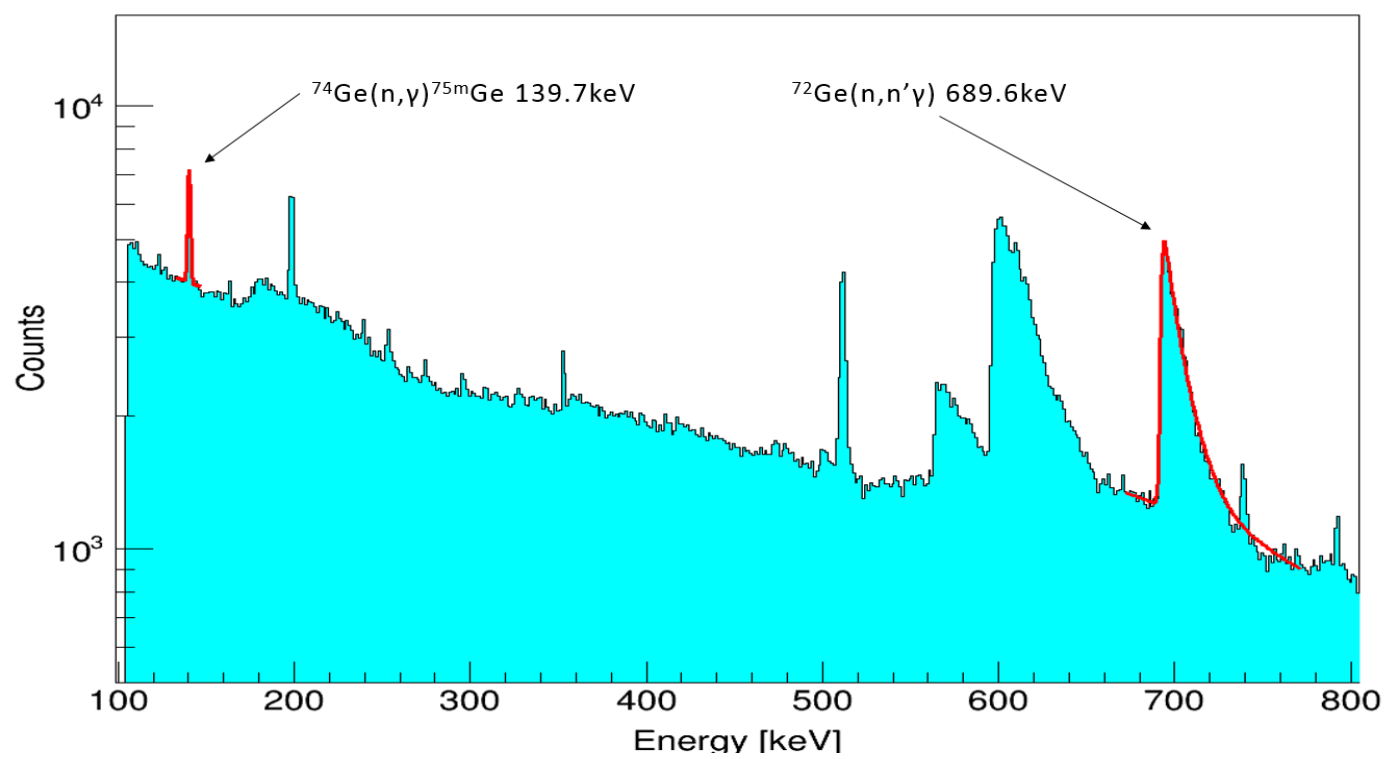

Figure 13. An example of HPGe spectrum from the measurements. $139.7 \mathrm{keV}$ gamma-rays are from thermal neutron capture reactions on $74 \mathrm{Ge}$. The $689.6 \mathrm{keV}$ peak with a high energy tail is caused by fast neutron inelastic scatterings on $72 \mathrm{Ge}$. The $139.7 \mathrm{keV}$ peak was fitted with a Gaussian function while the 689.6keV peak was fitted with an exponentially modified Gaussian (EMG) function.

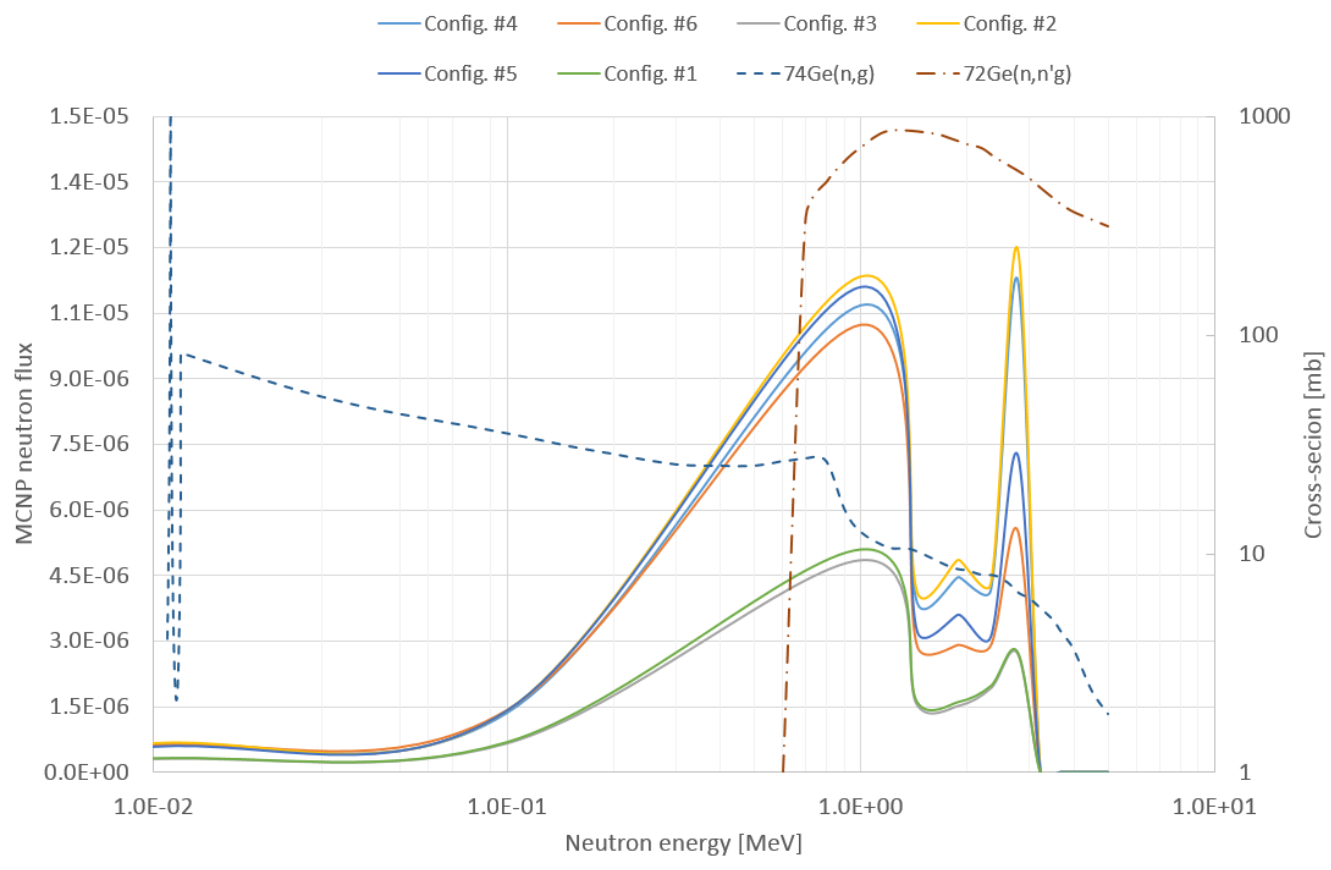

Figure 14. MCNP neutron fluxes of six configurations with no simulant. F4 tally was assigned to the HPGe crystal to obtain an energy distribution of incoming neutrons. Also, cross-sections of ${ }^{74} \mathrm{Ge}(\mathrm{n}, \gamma)$ and ${ }^{72} \mathrm{Ge}(\mathrm{n}, \mathrm{n}$ ' $\gamma)$ reactions are plotted in the unit of millibarn. 
Table 7. Summary of germanium 139.7 and $689.6 \mathrm{keV}$ net counts from the measured background spectra. Average values from two independent background spectra were adopted for each configuration. $N_{\text {total }}$ and $N_{\text {fast }}$ from MCNP results were calculated as discussed in section 6.

\begin{tabular}{|c|c|c|c|c|c|}
\hline \multirow{2}{*}{$\begin{array}{c}\text { Configuration \# } \\
\text { Simulant }\end{array}$} & Experiment & MCNP & Experiment & MCNP \\
\cline { 3 - 6 } & & Ge $139.7 \mathrm{keV}$ & $\begin{array}{c}N_{\text {total }} \\
{\left[\# / \mathrm{cm}^{2} / \mathrm{nps}\right]}\end{array}$ & Ge $689.6 \mathrm{keV}$ & $\begin{array}{c}N_{\text {fast }} \\
{\left[\# / \mathrm{cm}^{2} / \mathrm{nps}\right]}\end{array}$ \\
\hline 4 (wedge + small W) & Background & 6341 & $3.82 \mathrm{E}-05$ & 60801 & $3.44 \mathrm{E}-05$ \\
\hline 6 (wedge + large W) & Background & 4741 & $2.82 \mathrm{E}-05$ & 38490 & $2.45 \mathrm{E}-05$ \\
\hline 3 (wedge + medium W) & Background & 3224 & $1.47 \mathrm{E}-05$ & 23932 & $1.26 \mathrm{E}-05$ \\
\hline 2 (pyramid + small W) & Background & 6501 & $4.05 \mathrm{E}-05$ & 60795 & $3.66 \mathrm{E}-05$ \\
\hline 5 (pyramid + large W) & Background & 4725 & $3.18 \mathrm{E}-05$ & 42535 & $2.83 \mathrm{E}-05$ \\
\hline 1 (pyramid + medium W) & Background & 3108 & $1.53 \mathrm{E}-05$ & 27655 & $1.31 \mathrm{E}-05$ \\
\hline
\end{tabular}
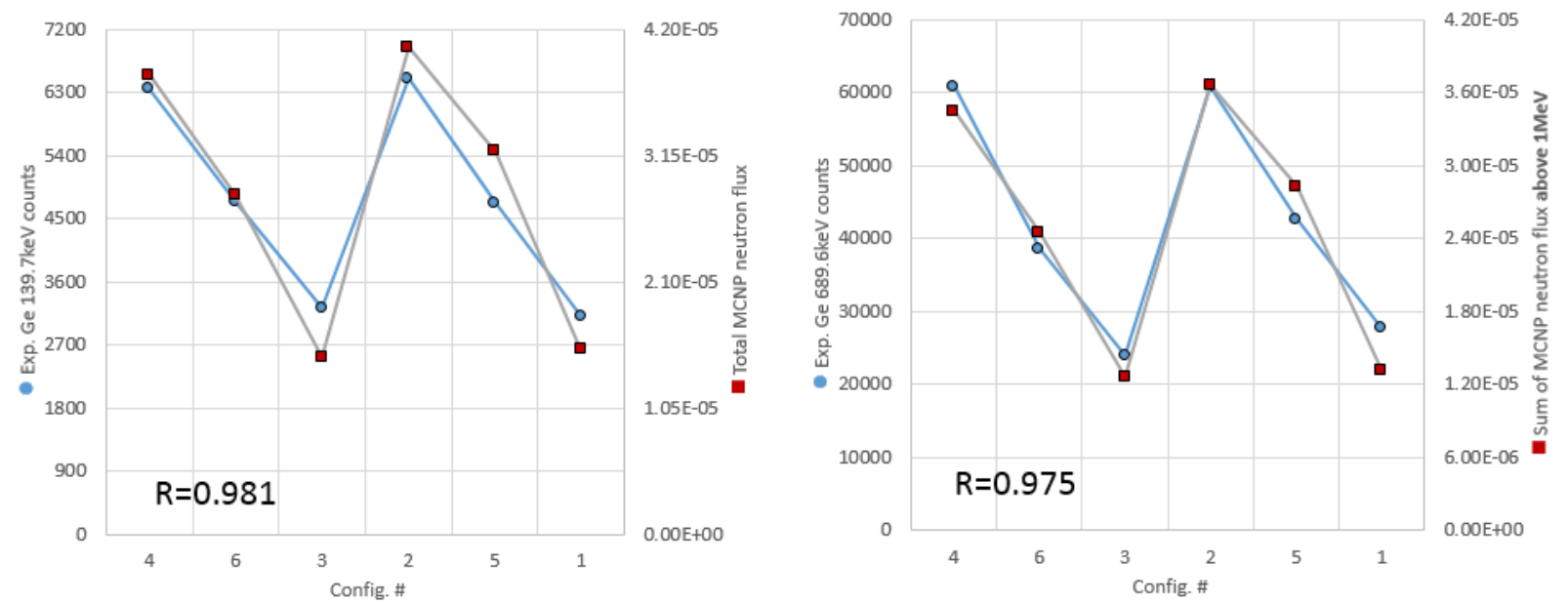

Figure 15. (Top) net counts of $139.7 \mathrm{keV}$ from ${ }^{74} \mathrm{Ge}(\mathrm{n}, \gamma)$ reaction were compared to $N_{\text {total }}$. (Bottom) net counts of $689.6 \mathrm{keV}$ from ${ }^{72} \mathrm{Ge}(\mathrm{n}, \mathrm{n}$ ' $\gamma)$ reaction were compared to $N_{\text {fast }}$. 

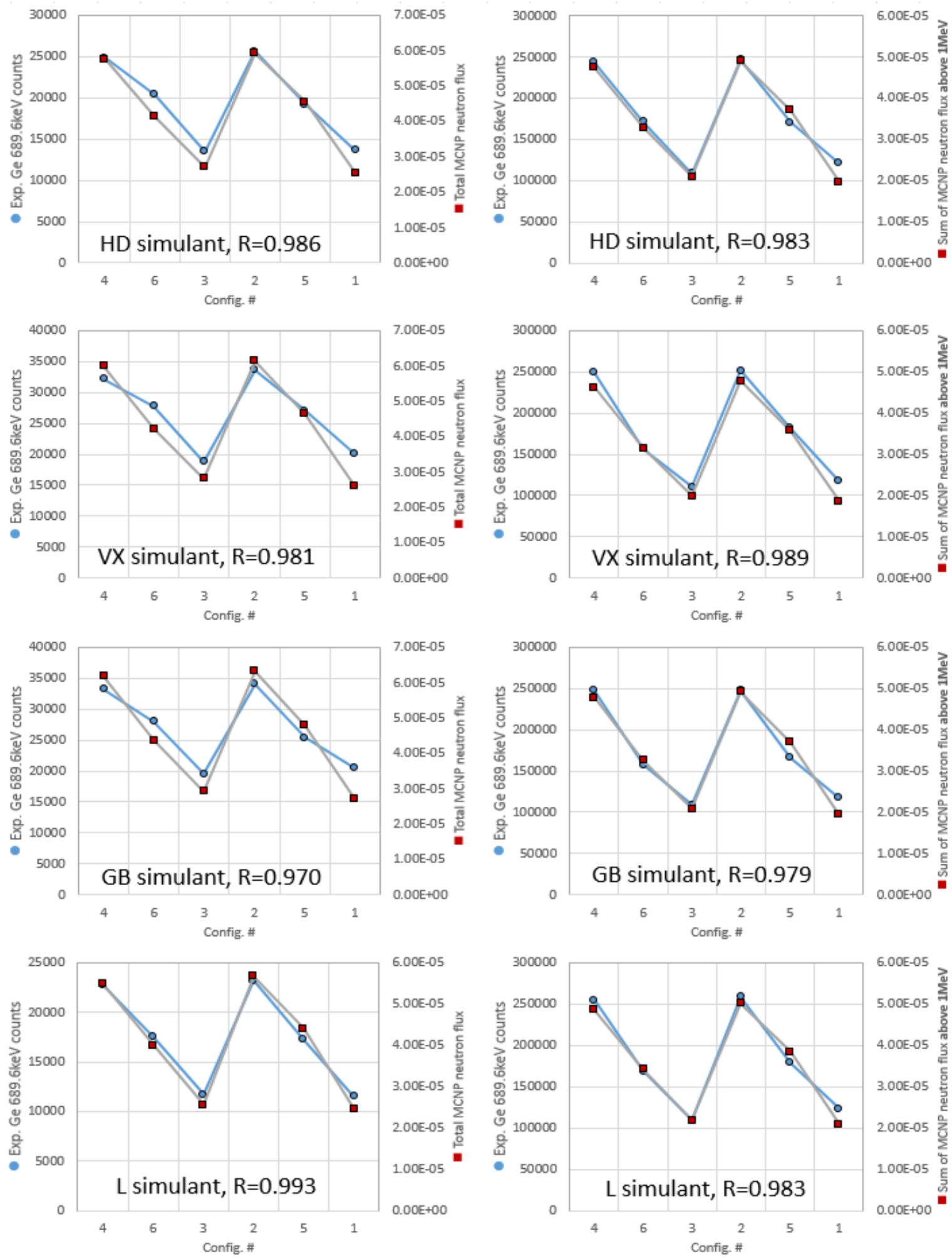

Figure 16. (Left column) net counts of $139.7 \mathrm{keV}$ were compared to $N_{\text {total }}$ for each simulant. (Right column) net counts of $689.6 \mathrm{keV}$ were compared to $N_{\text {fast }}$ for each simulant. Correlation coefficient of a pair of the data sets in each plot is also shown. Overall, there is strong linear proportionality between MCNP results and experimental data. 

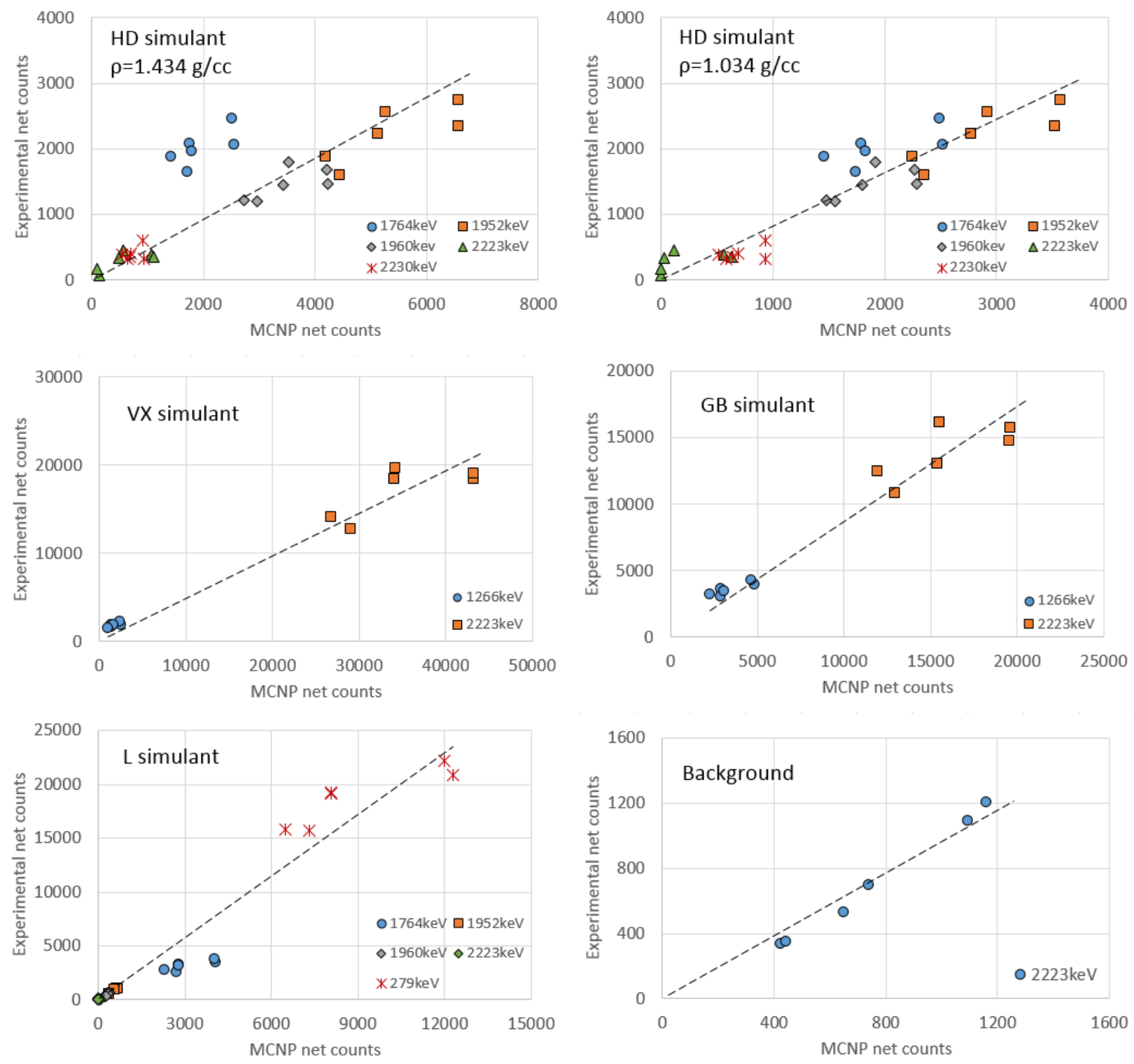

Figure 17. Experimental data versus MCNP results. MCNP results were normalized by each simulant's scale factor as discussed in section 5. The dotted lines are drawn to visualize positive linear relationships between MCNP results and experimental data. Two plots of The HD simulant with different densities are shown as an example of how simulant density affects MCNP results. Overall, there is a linear relationship between MCNP results and experimental data. 


\section{REFERENCES}

[1] J. Nikolov, et al., Applicability of the Ge(n, $\gamma)$ reaction for estimating thermal neutron flux, Phys. Procedia, 59 (2014), pp. 71-77 